\title{
Fuel Motion Monitoring System at TREAT - FY2017 Instrumentation Summary
}

D. L. Chichester, S. M. Watson, J. T. Johnson, J. D. Hix, S. J. Thompson, and J. L. Schulthess

September 2017

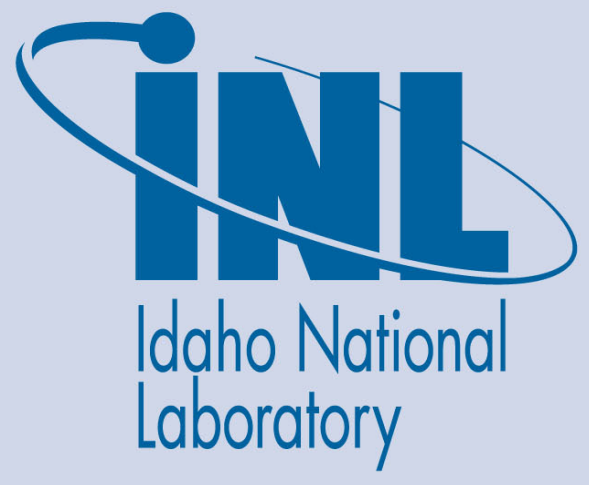

INL is a U.S. Department of Energy National Laboratory operated by Battelle Energy Alliance 


\section{DISCLAIMER}

This information was prepared as an account of work sponsored by an agency of the U.S. Government. Neither the U.S. Government nor any agency thereof, nor any of their employees, makes any warranty, expressed or implied, or assumes any legal liability or responsibility for the accuracy, completeness, or usefulness, of any information, apparatus, product, or process disclosed, or represents that its use would not infringe privately owned rights. References herein to any specific commercial product, process, or service by trade name, trade mark, manufacturer, or otherwise, do not necessarily constitute or imply its endorsement, recommendation, or favoring by the U.S. Government or any agency thereof. The views and opinions of authors expressed herein do not necessarily state or reflect those of the U.S. Government or any agency thereof. 


\section{Fuel Motion Monitoring System at TREAT - FY2017 Instrumentation Summary}

D. L. Chichester, S. M. Watson, J. T. Johnson, J. D. Hix, S. J. Thompson, and J. L. Schulthess

September 28, 2017

Idaho National Laboratory

Idaho Falls, Idaho 83415

http://www.inl.gov

Prepared for the U.S. Department of Energy Office of Nuclear Energy 


\section{ACKNOWLEDGEMENT}

We would like to thank the people at the TREAT facility for their ongoing support and encouragement, working with us to bring equipment out to the facility and install it. In particular, we wish to thank Brad Heath, Wynn Monk, Stuart Bondurant, Carl Bailey, Dan Wilcock, and J. R. Biggs. We'd also like to acknowledge and thank Scott Reed and Reed Carlson for their efforts related to returning the electromechanical system to operation. 


\section{EXECUTIVE SUMMARY}

This document is a report summarizing FY2017 efforts related to the Transient Reactor Test Facility (TREAT) Fuel Motion Monitoring System, "hodoscope," refurbishment campaign. TREAT is an air-cooled, graphite-moderated, heterogeneous test facility designed to evaluate reactor fuels and structural materials under conditions that simulate various types of transient overpower and under-cooling situations in a nuclear reactor. Fuel meltdowns, metal-water reactions, thermal interaction between overheated fuel and coolant, and the transient behavior of ceramic fuel for high-temperature systems can be studied. A key instrument that monitors fuel motion as these events take place is the fast neutron hodoscope. The hodoscope is designed to allow for pre-, during-, and posttransient imaging of fuel within a test loop in the center of the reactor core. The preferred technique for imaging is detection of fast neutrons produced by fission in the test fuel, travelling unmoderated through an empty row of core fuel assemblies and then through a multi-slot collimator to a 360-detector array. Due to the age of the instrument, a refurbishment of the hodoscope's fast neutron detection capability is necessary to support real-time, fuel-position experimental data collection. One particular challenge in this regard is related to the refurbishment of the fast-neutron-sensing detector elements in the detector array. This report summarizes major activities and accomplishments related to the FMMS refurbishment in FY2017. Of particular note was work related to identifying options for new proton recoil scintillator wafer and initial examination of the TREAT proton recoil proportional counter detectors. 


\section{FORWARD}

The TREAT facility is a special purpose reactor that is designed to execute experiments associated with nuclear reactor research and development. The reactor can accommodate experiment loops containing nuclear reactor fuel and material samples. The reactor can subject the test samples to off-normal test conditions. The experiment loop is usually heavily instrumented and a significant amount of test data must be collected and retained for analysis. The Hodoscope is a unique instrument designed to examine and record the movement of the fuel contained in the experiment loop in real time during transient conditions when fuel damage is produced. It is capable of examining movement through an optically opaque experiment loop with opaque coolant. Post irradiation examination performed on the experiment loops provides physical evidence of the transient end results while the Hodoscope provides information on how it got there. Following each test the Hodoscope will provide data to other data processing equipment for post-test analysis and report generation. Hodoscope generated data consists mainly of count rate data from fast neutron detectors. Conversion of count rate to images and reports requires post-transient analysis supported by modeling and pre-transient experimental activities. 


\section{CONTENTS}

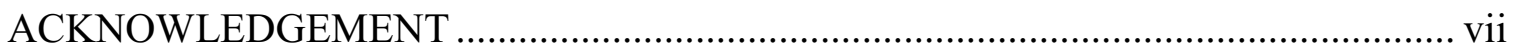

EXECUTIVE SUMMARY …………………………......................................... ix

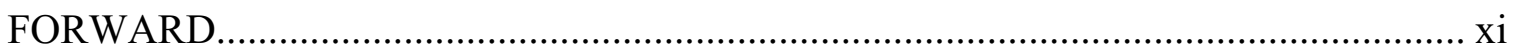

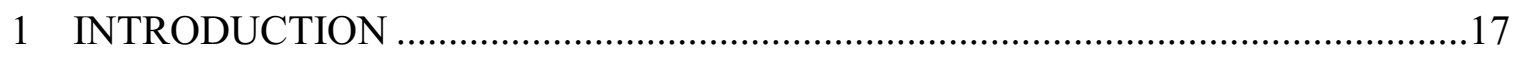

1.1 Background Information - TREAT FMMS....................................................17

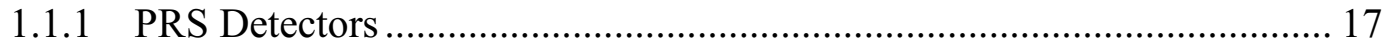

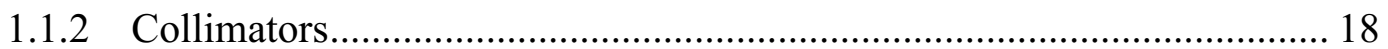

1.1.3 Limited View vs. Full View Imaging ................................................... 18

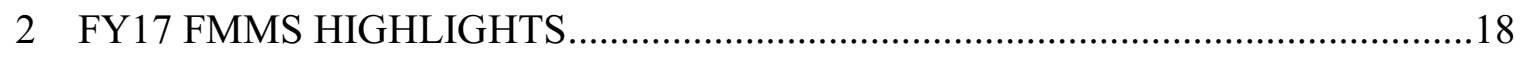

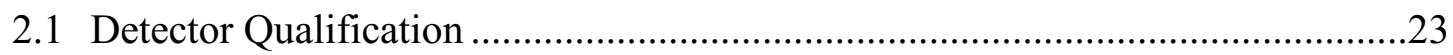

2.2 Qualification Methodology ……………………..............................................29

3 DATA ANALYSIS / VISUALIZATION SOFTWARE ………………......................32

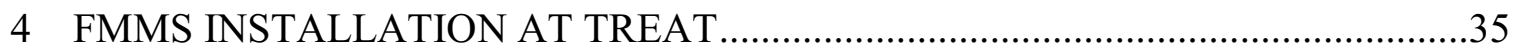

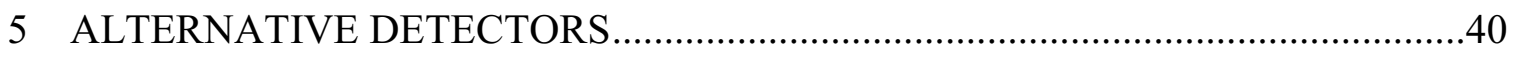

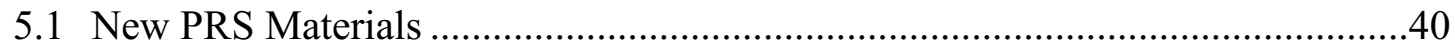

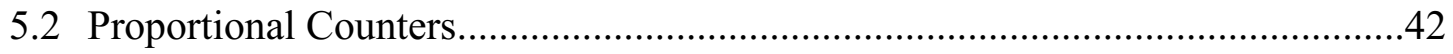

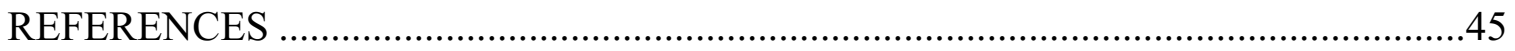




\section{FIGURES}

Figure 1 Front view of the 96-channel FMMS with source positioner located in RSDL.............. 19

Figure 2 Close-up view of the prototype FMMS detector array located in RSDL........................ 20

Figure 3 Rearview of the 96-channel FMMS with source positioner, located in RSDL.............. 21

Figure 4 The FMMS data acquisition system cabinet with all cabling connected. From the top of the cabinet: DAS control computer, pull out drawer with monitor and keyboard, Ethernet hub, Wiener custom chassis with digitizer, and high voltage cards.

Figure 5 This photograph shows the test stations used to qualify PMTs (Test Station \#1) and PMT/PRS assemblies (Test Station \#2) along with the digitizer and high voltage modules used to with the stations.

Figure 6 Individual components that make up a PRS assembly

Figure 7 Optical output of a typical qualified PRS, showing both painted and unpainted conditions. Note the increase in photon emission after application of scintillator paint.

Figure 8 Photomultiplier tubes were placed in phenolic shrouds and tested inside a light tight enclosure using a normalized Gaussian-distributed 420-nm LED. It was pulsed and directly coupled to an acrylic waveguide held in the center of the test station (\#1).

Figure 9 The graph on the left shows the plot of the measured light distribution as a function of test position inside the PMT test enclosure. The table on the right shows the calculated correction factors used to obtain an evenly distributed light source.

Figure 10 Uncompensated (left) and temperature-compensated (right) PMT response versus temperature for seven PMTs.

Figure $11 \mathrm{~A}$ set of PRS/PMT assemblies configured around a ${ }^{252} \mathrm{Cf}$ source inside Test Station \#2, to offer evenly distributed neutron flux for PSD optimization and detector-to-detector normalization.

Figure 12 Overall qualification methodology and collected data per process for each PRS, PMT, and PRS/PMT assembly.

Figure 13 A schematic demonstrating how the PSD value is calculated from a digitized detector pulse (the pulse has been inverted for display).

Figure 14 Individual PSD maps assigned per detector assembly based on PMT and PRS

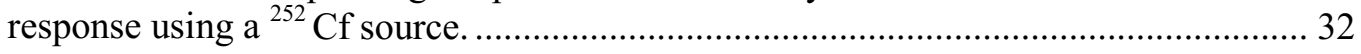

Figure 15 A screenshot of the FMMS Data Viewer application.

Figure 16 An expanded view of the TREAT reactor power profile plot and data playback controls section of the FMMS Data Viewer user interface

Figure 17 An expanded view of the 1-D and 2-D visualization sections of the FMMS

Data Viewer user interface.

Figure 18 FMMS engineers load and secure the TREAT hodoscope for shipment..................... 36

Figure 19 PRS/PMT assemblies ready for installation into the hodoscope at TREAT 
Figure 20 A view inside the light-tight PRS detector cabinet on the hodoscope at the TREAT north port. Detector assemblies are aligned with rear collimator channels.

Figure 21 FMMS engineers and a TREAT Instrumentation and Control technician work side-by-side to ensure proper cable routing and dressing during the LV FMMS installation

Figure 22 A view of the final connections made to the FMMS DAS at TREAT......................... 38

Figure 23 Installation of the DAS, ready for testing.

Figure 24 Various scintillation materials used in the search for a replacement for the PRS detectors.

Figure 25 Optical output response of various materials tested, using a 300-nm light source for excitation. Note the $20 \% \mathrm{ZnS}(\mathrm{Ag})$ mixture in optical epoxy

demonstrates the best result of the new candidate sensors.

Figure 26 X-ray image of Reuter-Stokes (model RS-P5-0808-204) PRPC.

Figure 27 Reuter-Stokes (model RS-P5-0808-204) under test inside acrylic HV shield.

Figure 28 Initial experimental set up for PRPC testing. Note the use of the $+8 \mathrm{kV}$, fourchannel high voltage supply since the bias turn on voltage was stated as $+5,600$ $\mathrm{V}$. 


\section{Fuel Motion Monitoring System at TREAT - FY2017 Instrumentation Report}

\section{INTRODUCTION}

The Fuel Motion Monitoring System (FMMS, hodoscope) at the Transient Reactor Test Facility (TREAT) provides unique data on the dynamic behavior of nuclear fuel under extreme accident conditions as a part of the TREAT Transient Fuel Science Program. The TREAT Transient Fuel Science Program is an applied science research and development activity focused towards studying the behavior of nuclear fuel under transient nuclear accident conditions to gain insight into how and why fuel fails. The goal of this program is to use this knowledge to improve our understanding of the safety margins of current and future nuclear fuel systems and to develop new fuel systems that extended these margins.

\subsection{Background Information - TREAT FMMS}

The FMMS was placed into standby when the TREAT reactor ceased operation in 1994. In parallel with the current TREAT reactor restart activities work is also underway to refurbish the FMMS and return it to operation. The plan for refurbishing the FMMS has three phases.

- Phase I - Establish a limited field of view imaging capability reusing the 1.2-m field-of-view collimator and other existing components, to the extent possible, with the goal of using the FMMS proton recoil scintillator (PRS) detectors to establish 96 channels of simultaneous, real-time data acquisition.

- Phase II - Using lessons learned from phase 1, expand the system to accommodate a full array of 360 imaging channels using PRS detectors.

- $\quad$ Phase III - Refurbish and deploy 360 channels of proton recoil proportional counter (PRPC) detectors.

\subsubsection{PRS Detectors}

The FMMS PRS detectors are composed of a mixture of silver-activated zinc sulfide $(\mathrm{ZnS}(\mathrm{Ag}))$ grains dispersed in a rectangular Lucite wafer. Fast neutrons incident on this wafer scatter with hydrogen, which produces recoil protons that travel for a short range within the Lucite before slowing down and stopping in the matrix. During this slowingdown process these recoil protons can interact with the $\mathrm{ZnS}(\mathrm{Ag})$ grains, exciting higherenergy quantum states in the $\mathrm{ZnS}$ molecule, which subsequently de-excite with the emission of visible photons (blue, $\sim 450 \mathrm{~nm}$ ). The wafers are coupled to two hemicylindrical Lucite wave guides. This scintillation assembly is coupled to a 
photomultiplier tube (PMT) which detects the viable scintillation photons. The PRS/PMT assemblies are placed in phenolic shrouds that serve as both a way to minimize ambient light contamination in the PMT and to offer a means of rotational alignment between the PRS and the FMMS's collimator rectangular channel slits. The detectors are housed within a large metal cabinet which serves as a near-light-tight enclosure cabinet.

\subsubsection{Collimators}

Collimation in the FMMS is accomplished with front and rear collimators. The front collimator is a wedge-shaped horizontal slit placed within the TREAT reactor's north beam-port opening. The rear collimator is a stack of slotted steel plates that extends partially into the TREAT reactor's north beam port. This collimation configuration blocks uncollimated neutrons coming from the TREAT core from impinging on the FMMS fastneutron detectors. The rear collimator rests on the FMMS platform, along with the detector cabinets, and is translatable to allow alignment of the collimator with a test capsule or fuel undergoing testing.

\subsubsection{Limited View vs. Full View Imaging}

Originally, a total of seven hundred twenty (720) fast neuron detectors, 360 PRS detectors and 360 PRPC detectors, were mounted in two separate detector cabinets on the FMMS platform. The PRPC detectors were closest to the collimator, with the array of PRS detectors directly behind the PRPC detectors. The 720 detector configuration is defined as the full view FMMS and any configuration that provides less than 720 detectors is defined as the limited view FMMS.

\section{FY17 FMMS HIGHLIGHTS}

The INL FMMS scientific team assembled and tested a fully-operational prototype 96channel limited view FMMS in INL's Radiation Detection Systems Laboratory (RDSL). The detector array components and electronics hardware, in a 16-channel configuration, were first documented in a memo (CCN 238491) sent to Dr. Dan Wachs on June 29, 2016 (revised July 12, 2016). The hardware described in CCN 238491 was expanded from 16 to 96 channels, along with 16 spares, at the RDSL in the first half of fiscal year (FY) 2017; this equipment was taken from RDSL and installed at TREAT in the second half of FY2017. Work related to installing the equipment at TREAT included labeling, routing, and connecting 192 cable assemblies in the PRS detector cabinet and expansion of the FMMS LabView software used to control 96 individual channels. This software development also included creation of an automated approach for calibrating and normalizing the PRS detector array. In addition, qualification of 112 detector components was also performed. Photographs of the overall FMMS system located in RDSL, prior to transport to TREAT, along with close ups of the detector array and the data acquisition system (DAS) cabinet are shown in Figure 1 through Figure 4. 


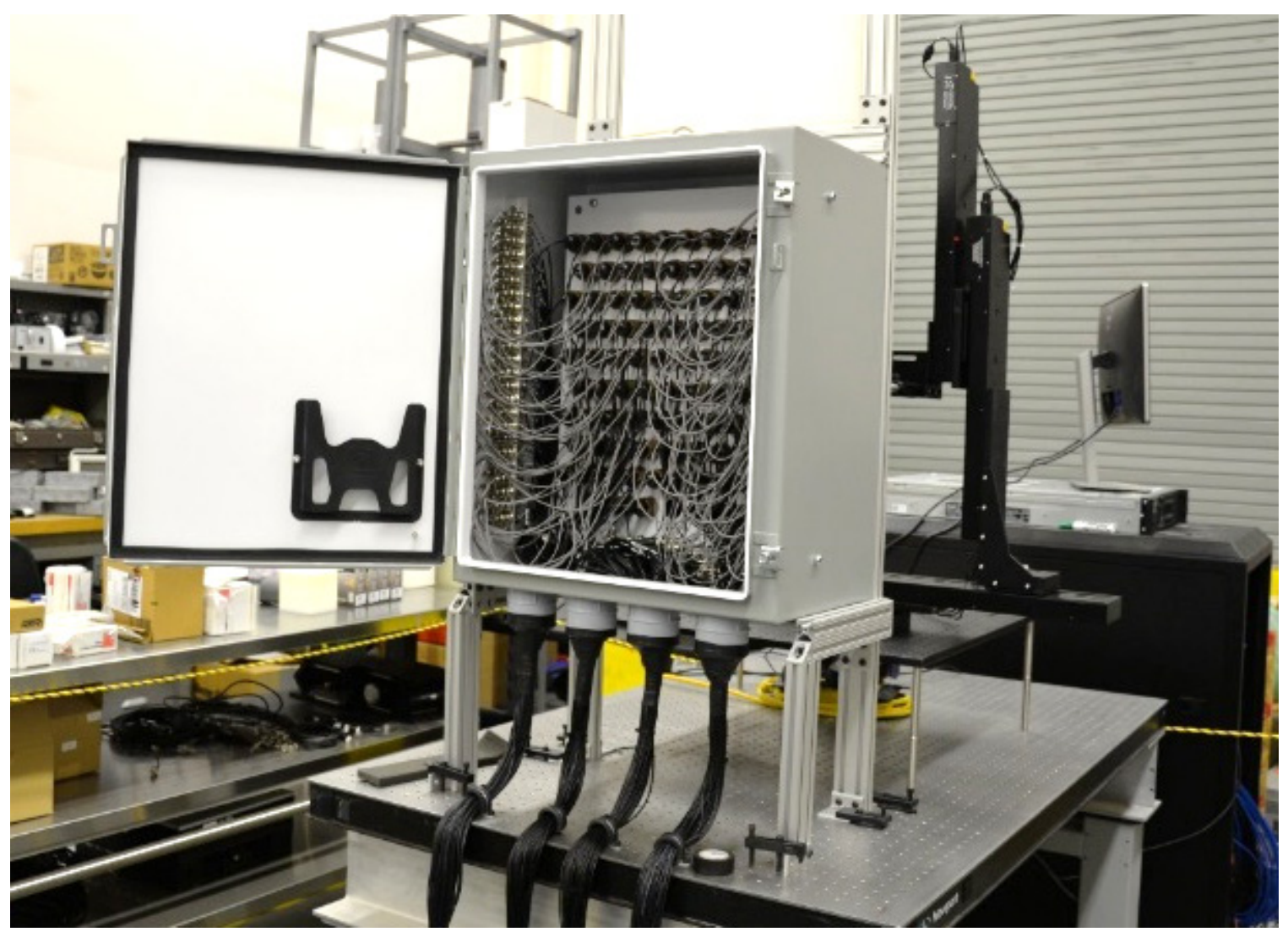

Figure 1 Front view of the 96-channel FMMS with source positioner located in RSDL. 


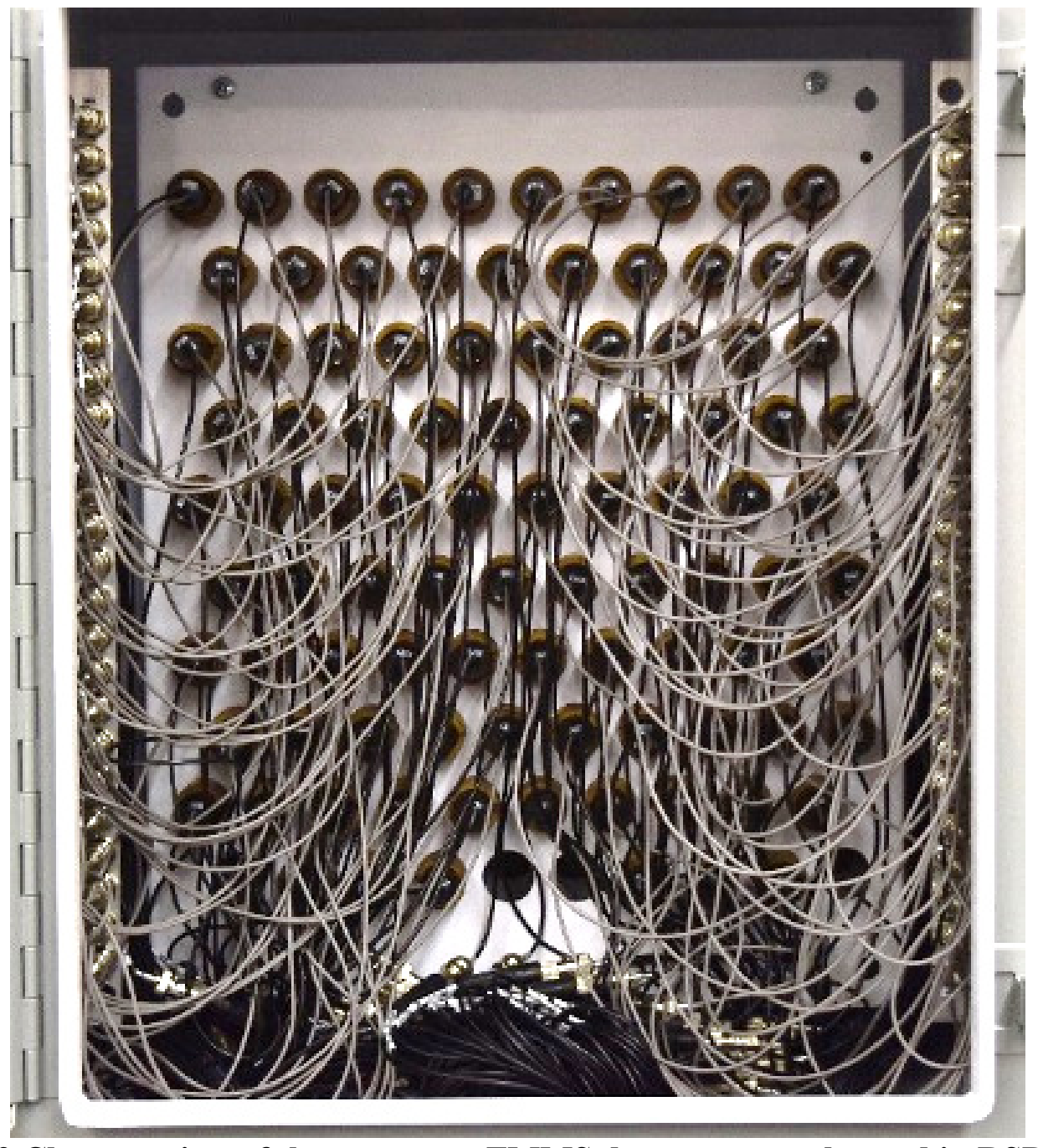

Figure 2 Close-up view of the prototype FMMS detector array located in RSDL. 


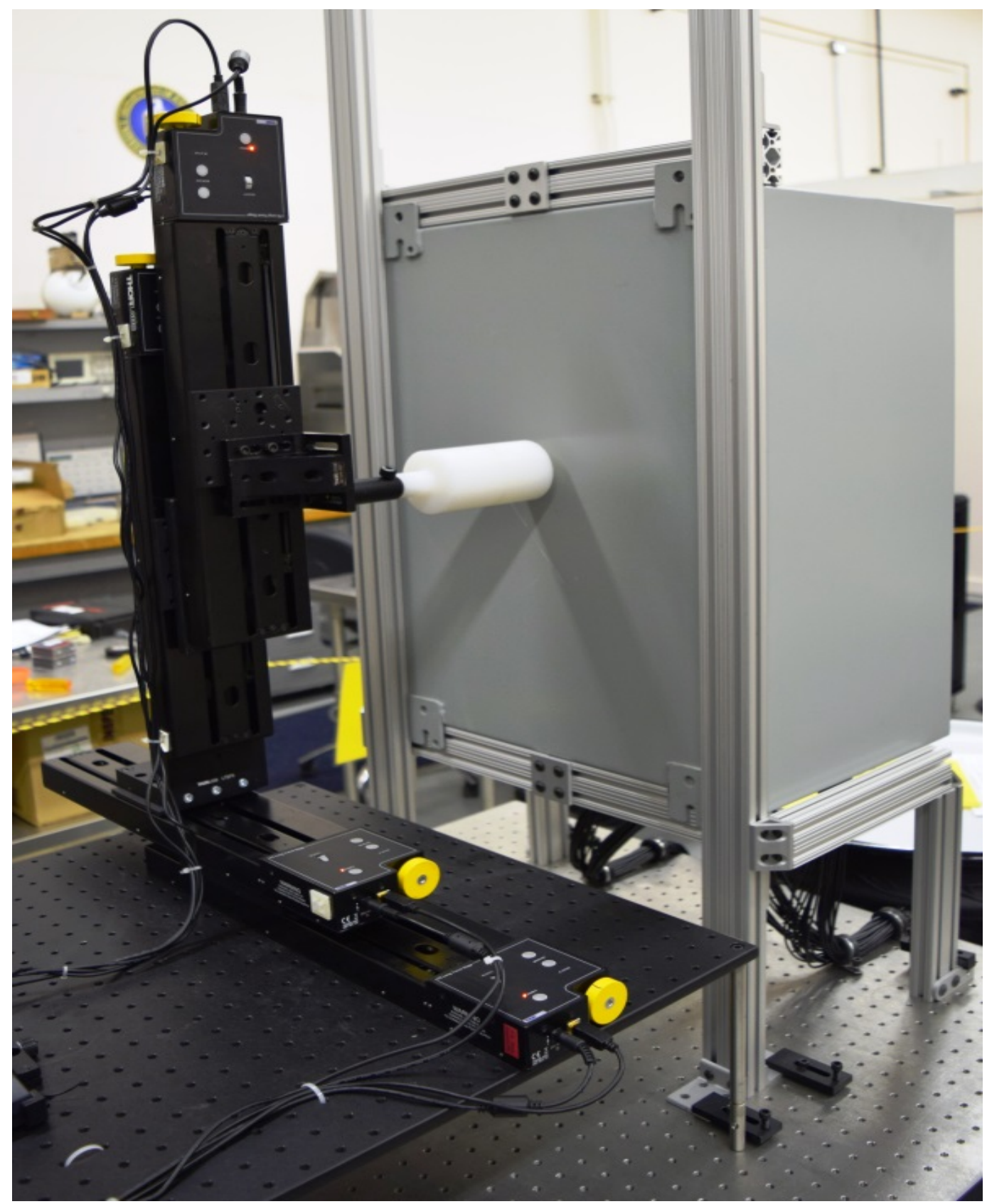

Figure 3 Rearview of the 96-channel FMMS with source positioner, located in RSDL. 


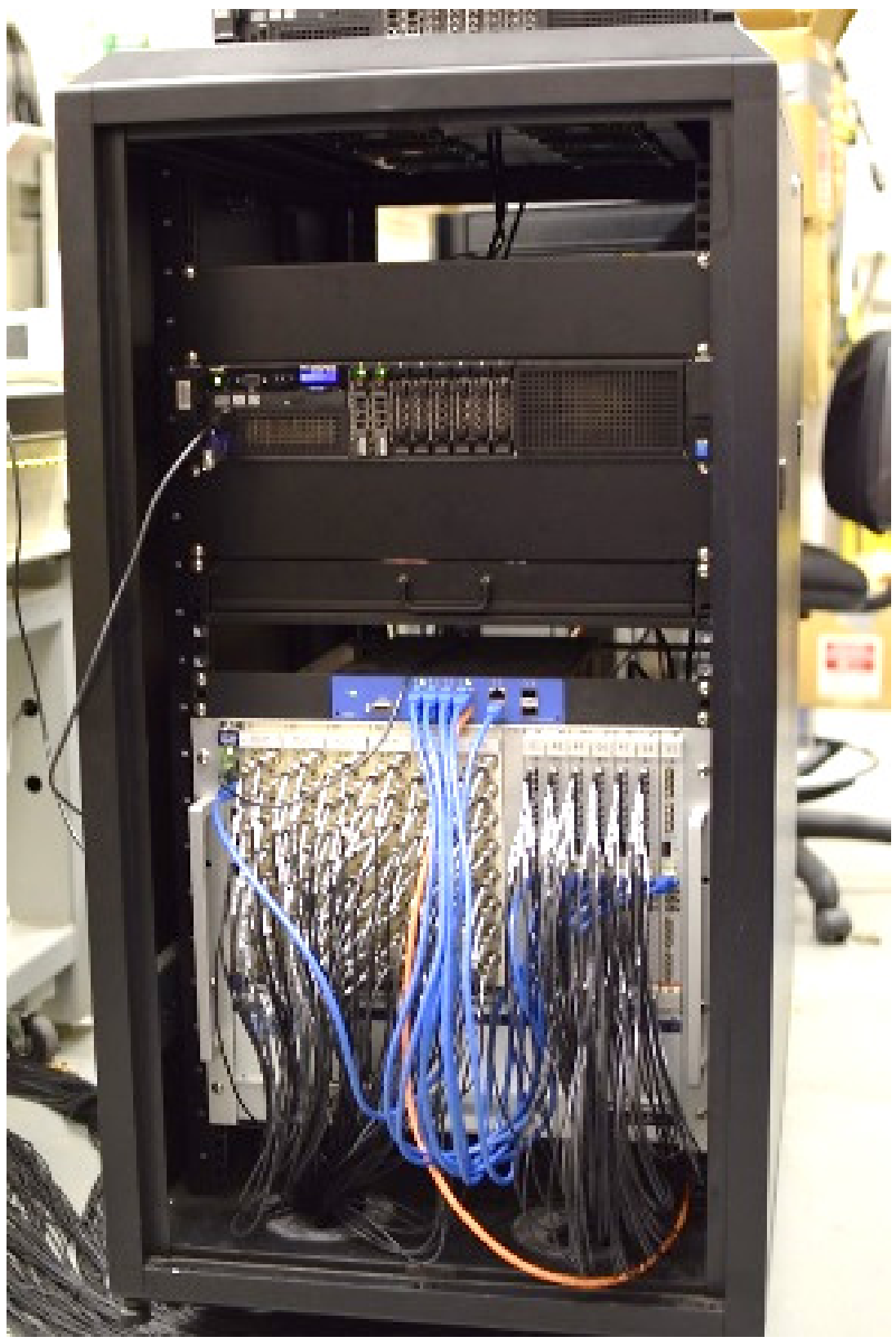

Figure 4 The FMMS data acquisition system cabinet with all cabling connected. From the top of the cabinet: DAS control computer, pull out drawer with monitor and keyboard, Ethernet hub, Wiener custom chassis with digitizer, and high voltage cards. 


\subsection{Detector Qualification}

To facilitate the qualification of detector components, two separate experimental test stations were designed and built to test each photomultiplier tube (PMT), proton recoil scintillator (PRS), and PMT/PRS assembly. This qualification was necessary to ensure components fell within a previously documented FMMS requirement to have a tolerance of $\pm 10 \%$ between individual PRS assemblies.

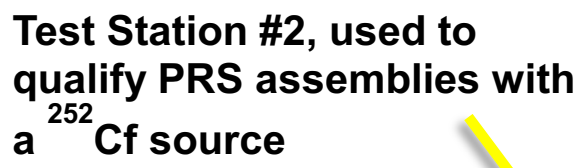

\section{Test Station \#1, used to qualify PMTs with an LED light source}

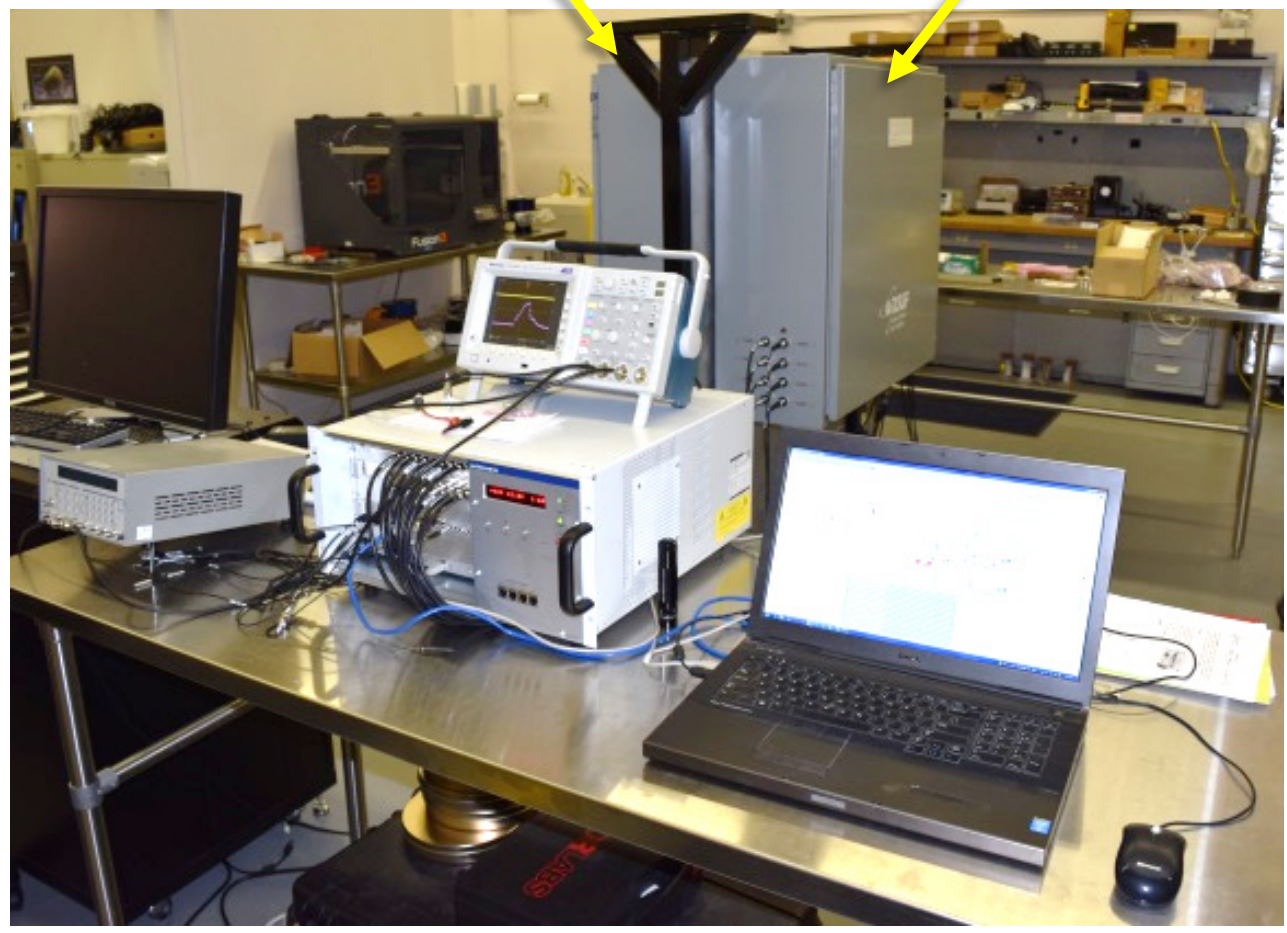

Figure 5 This photograph shows the test stations used to qualify PMTs (Test Station \#1) and PMT/PRS assemblies (Test Station \#2) along with the digitizer and high voltage modules used to with the stations.

The FMMS PRS detectors are composed of a mixture of silver-activated zinc sulfide $(\mathrm{ZnS}(\mathrm{Ag}))$ grains dispersed in a rectangular Lucite wafer. Fast neutrons incident on this wafer scatter with hydrogen, which produces recoil protons that travel for a short range within the Lucite before slowing down and stopping in the matrix. During this slowingdown process these recoil protons can interact with the $\mathrm{ZnS}(\mathrm{Ag})$ grains, exciting higherenergy quantum states in the $\mathrm{ZnS}$ molecule, which subsequently de-excite with the emission of visible (blue, $\sim 450 \mathrm{~nm}$ ) photons. The wafers are coupled to two hemicylindrical Lucite wave guides. This scintillation assembly is coupled to a photomultiplier tube (PMT) which detects the viable scintillation photons. The PRS/PMT assemblies are placed in phenolic shrouds that serve as both a way to minimize ambient light contamination in the PMT and to offer a means of rotational alignment between the PRS and the rectangular collimator channel slits. The detectors are housed within a large 
metal cabinet which serves as a near light-tight enclosure. The individual components that make up a PRS assembly are illustrated in Figure 6.

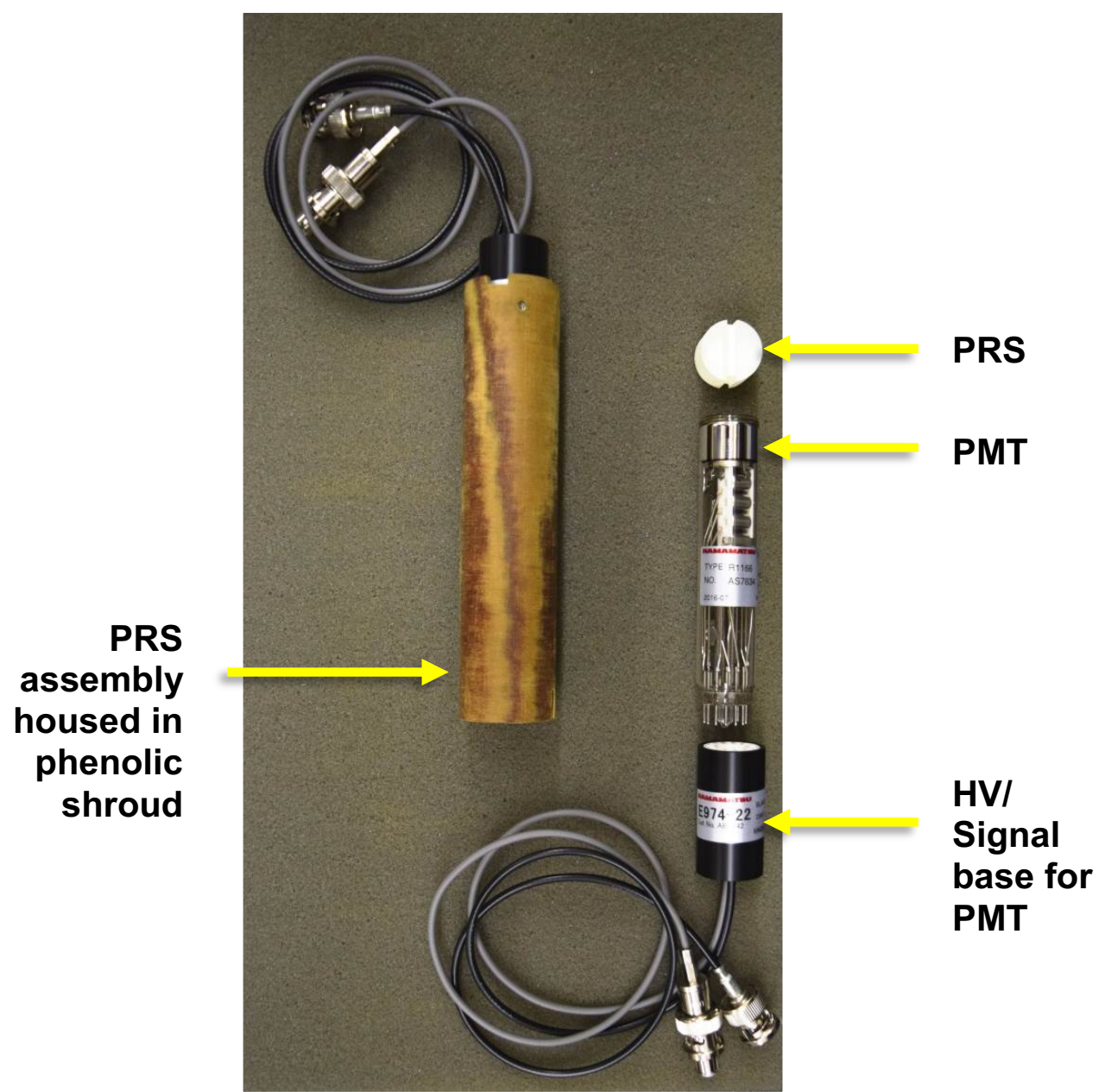

Figure 6 Individual components that make up a PRS assembly.

The initial down selection process began by visually inspecting each PRS recovered from TREAT for the qualities of symmetric shape during manufacture, clarity/adhesion of the Lucite waveguides to the $\mathrm{ZnS}(\mathrm{Ag}$ ) wafer, and the color of the $\mathrm{ZnS}(\mathrm{Ag}$ ) wafer, bright white being the benchmark. Down selected PRSs were qualified as a function of induced photon emission using a fiberized ultraviolet enhanced charge coupled device- (CCD-) based spectrometer. Spectral collection was accomplished using an integrating sphere; individual PRSs were placed inside the sphere and optical fibers were attached to offer both illumination and collection capability. The emission light source was a highly stable fiberized 280-nm light-emitting diode (LED). Two conditions were measured. First, the PRSs were stripped of original paint, cleaned with reagent grade alcohol, and measured as a function of wavelength output. This was the unpainted condition. Second, the PRSs were painted with an air brush using Eljen Technologies EJ-500 scintillator paint and measured again under the same conditions. This was the painted condition. The graph in Figure 7 shows both conditions. 


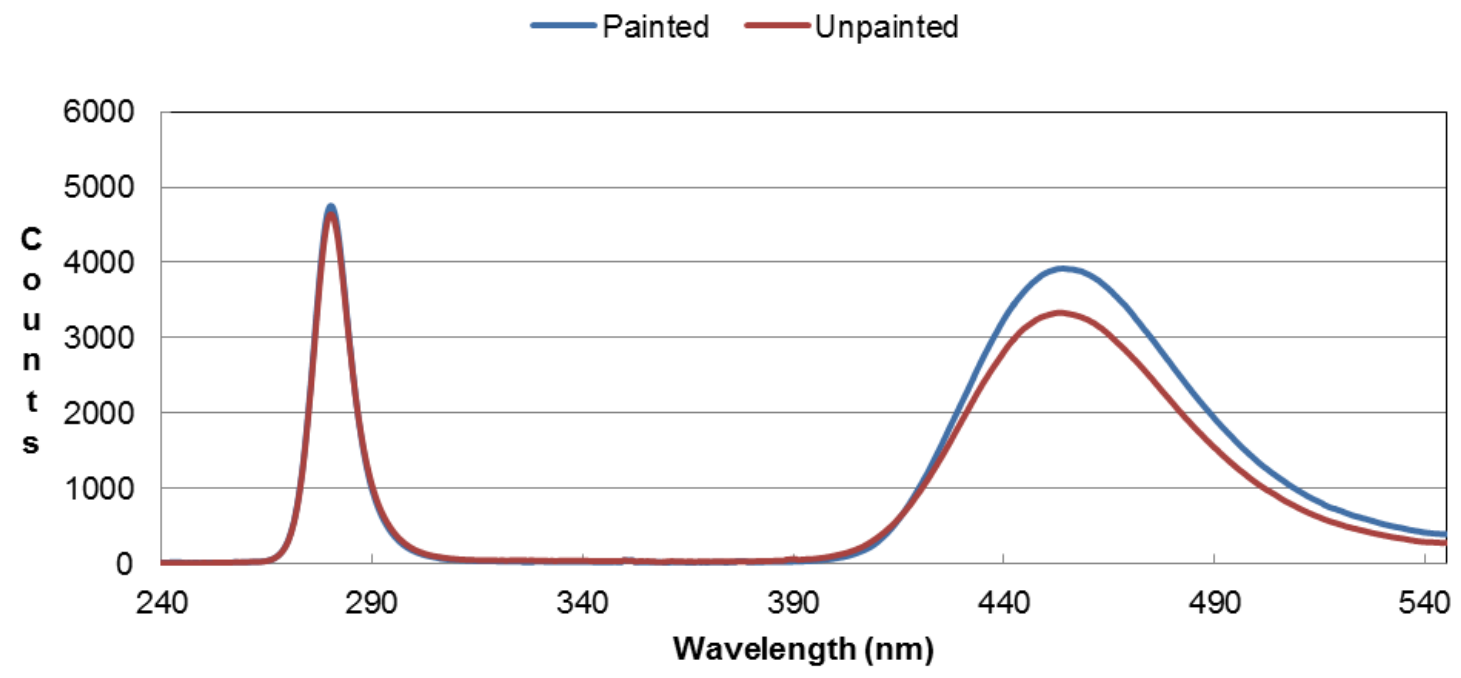

Figure 7 Optical output of a typical qualified PRS, showing both painted and unpainted conditions. Note the increase in photon emission after application of scintillator paint.

After the optical qualification of down-selected PMTs was completed, a methodology was developed to qualify both the PMTs and PMT/PRS assemblies. Photomultiplier tubes were placed in phenolic shrouds and tested inside a light tight enclosure using a normalized, Gaussian-distributed 420-nm LED that was pulsed and directly coupled to an acrylic waveguide held in the center of Test Station \#1. The bias voltage was swept across the span of recommended manufacturers operating voltages from minimum to maximum $(-500 \mathrm{~V}$ through $-1250 \mathrm{~V})$ using modular, automated high-voltage power supplies. The operational set up for testing the PMTs inside the enclosure is shown Figure 8. 


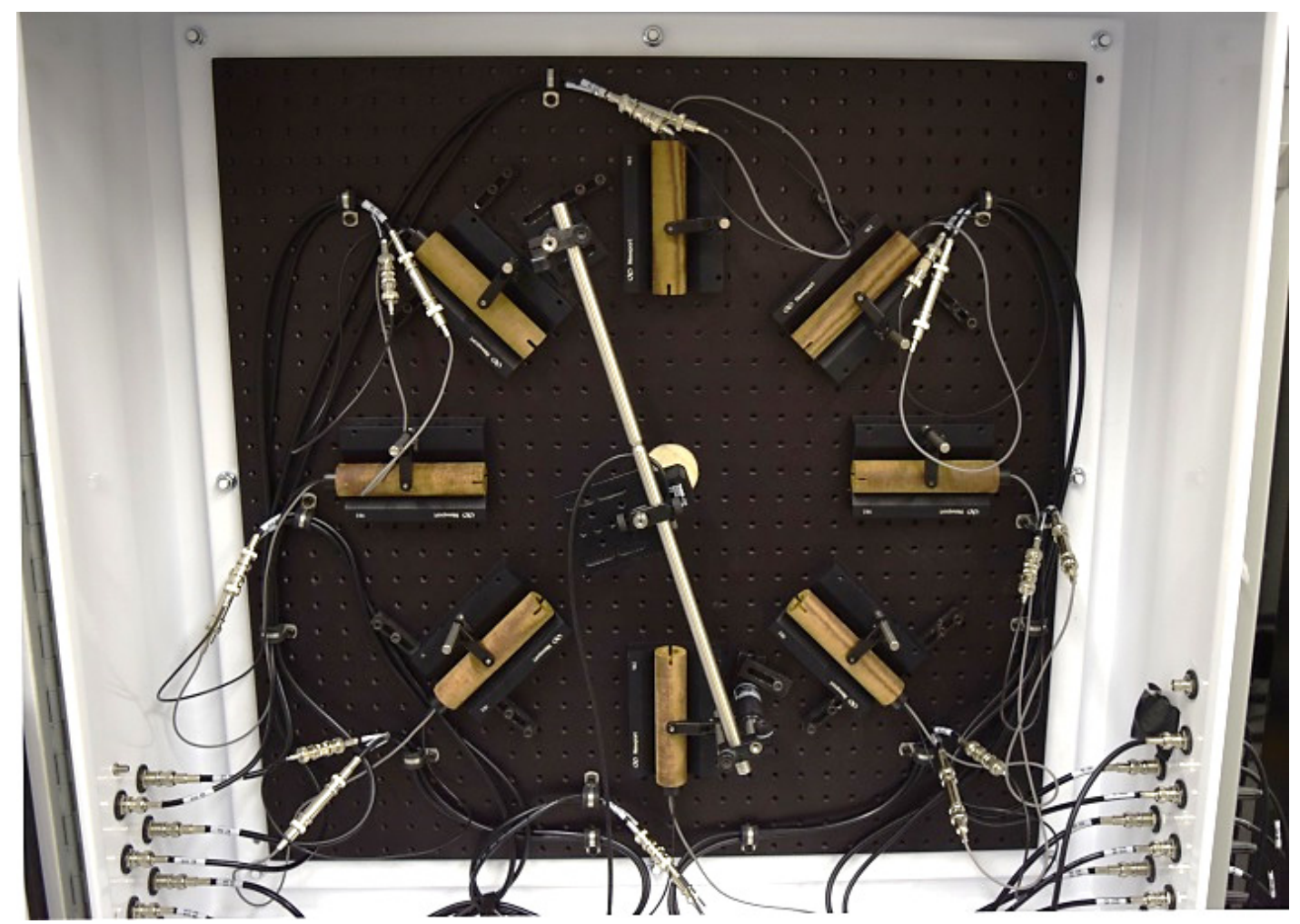

Figure 8 Photomultiplier tubes were placed in phenolic shrouds and tested inside a light tight enclosure using a normalized Gaussian-distributed 420-nm LED. It was pulsed and directly coupled to an acrylic waveguide held in the center of the test station (\#1).

Illumination variability of the 420-nm LED light source distribution system was verified by using a single PMT and recording light pulse data in each of the eight PMT test positions. This process was repeated with a different PMT to reduce statistical error. Data collected from this process is shown in Figure 9 on the left. To compensate for variability a light correction factor was calculated from the averaged output of two PMTs at coinciding positions. The light correction factor was then applied to data collected in real time, to obtain a normalized evenly distributed light source. The table on the right in Figure 9 shows the correction factors that were applied. 


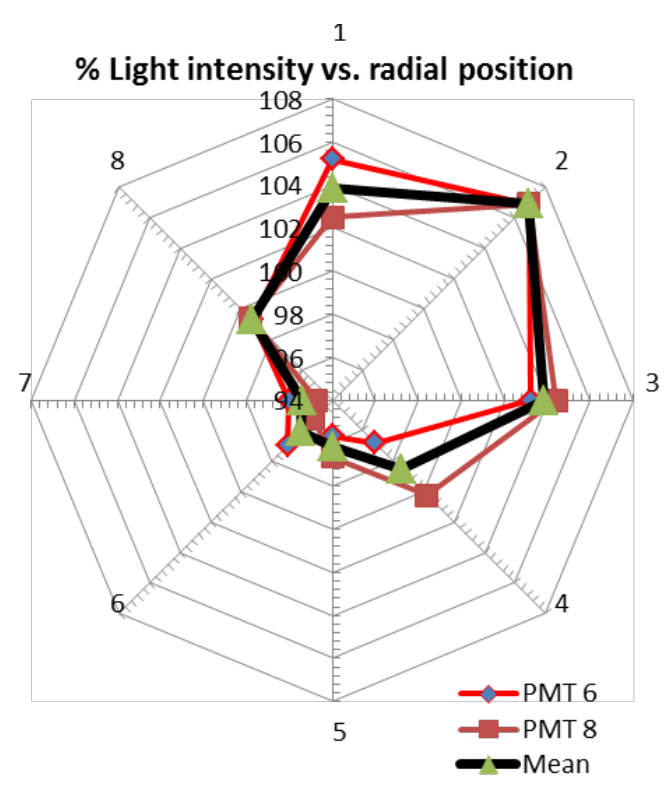

\begin{tabular}{|c|c|c|c|}
\hline \multicolumn{4}{|c|}{ Light Correction Factor } \\
\hline Position & $\begin{array}{l}\text { PMT } 6 \\
863 \mathrm{~V}\end{array}$ & $\begin{array}{l}\text { PMT } 8 \\
824 \text { V }\end{array}$ & Average \\
\hline 1 & 1.052 & 1.025 & 1.038 \\
\hline 2 & 1.069 & 1.069 & 1.069 \\
\hline 3 & 1.032 & 1.044 & 1.038 \\
\hline 4 & 0.968 & 1.002 & 0.985 \\
\hline 5 & 0.957 & 0.966 & 0.961 \\
\hline 6 & 0.969 & 0.952 & 0.961 \\
\hline 7 & 0.960 & 0.947 & 0.954 \\
\hline 8 & 0.993 & 0.994 & 0.993 \\
\hline
\end{tabular}

Figure 9 The graph on the left shows the plot of the measured light distribution as a function of test position inside the PMT test enclosure. The table on the right shows the calculated correction factors used to obtain an evenly distributed light source.

A PMT's performance is temperature dependent; the gain of a PMT is inversely proportional to temperature. The temperature from test to test varies, so in order to get consistent results PMT performance needs to be adjusted to account for temperature drift. A National Instruments USB-TC01 temperature probe was used to measure the temperature inside the test station. The temperature response was measured for eight different PMTs. A simple equation (Eq. 1) was then formulated to compensate PMT gain to a fixed temperature of 72 degrees Fahrenheit. The difference between compensated and uncompensated PMT performance is shown in Figure 10.

Compensated $=$ Uncompensated $+0.0879 *\left(P M T\right.$ Temperature $\left.-72^{\circ} F\right) \quad$ Eq. 1 

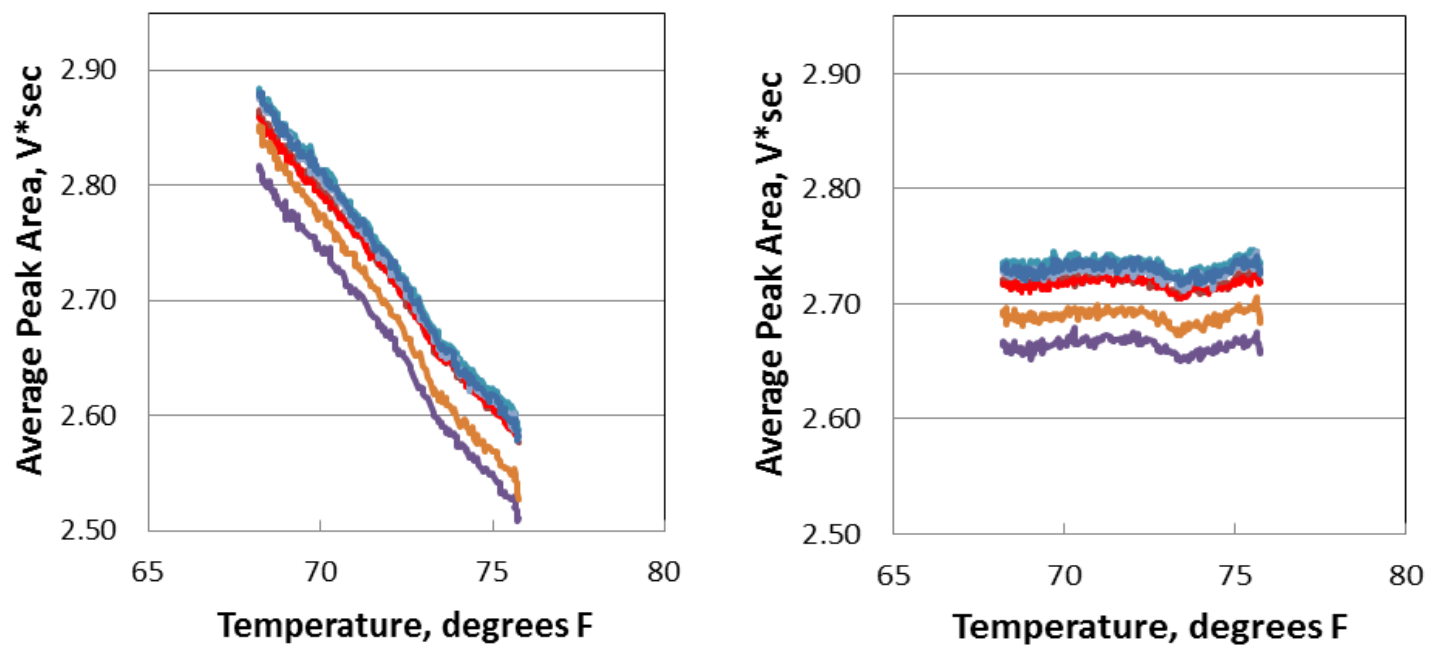

Figure 10 Uncompensated (left) and temperature-compensated (right) PMT response versus temperature for seven PMTs.
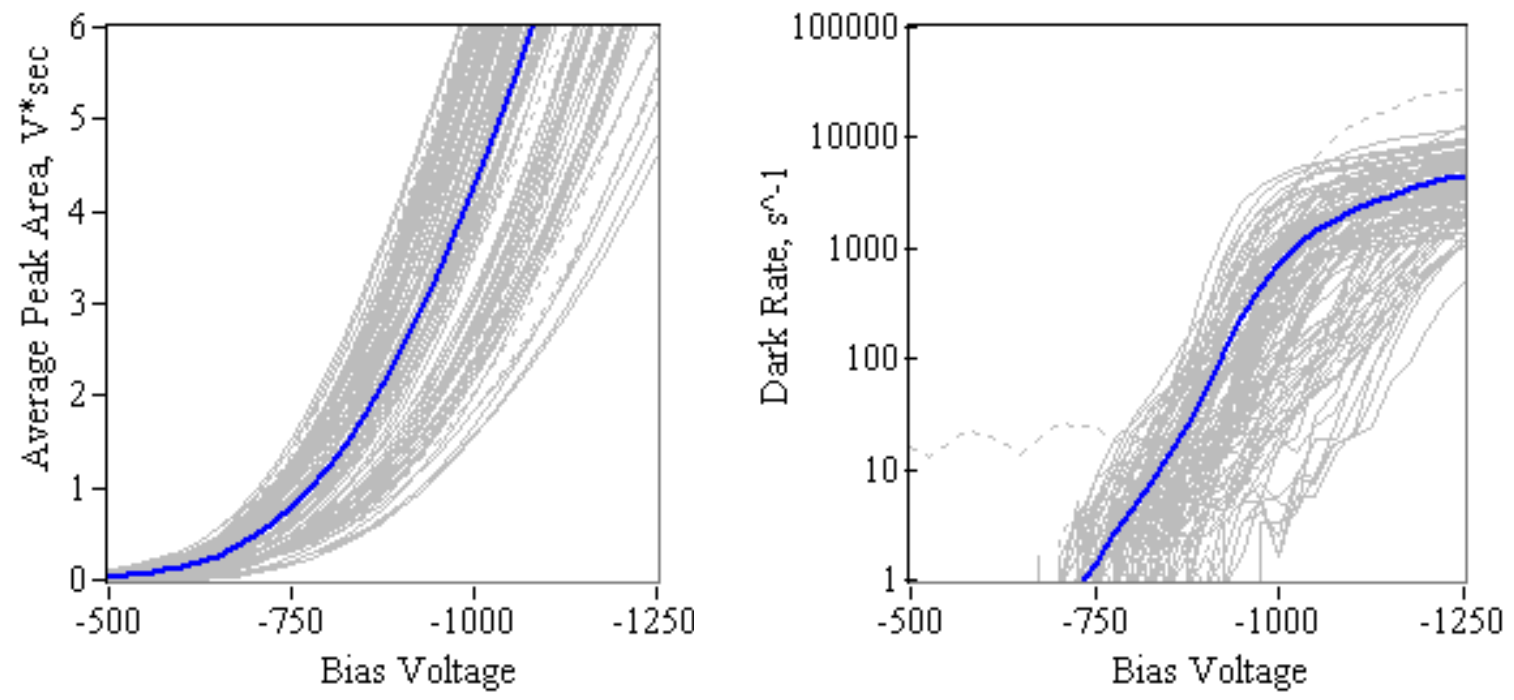

Figure 11 Voltage bias sweeps with the pulsed LED light source on (left) and off (right), showing PMT response and dark current.

The data collected from analyzing 112 PMTs as a function of temperature compensated voltage bias sweep is shown in Figure 11. The linear-scale graph on the left plots the average peak area of the PMT output pulse vs the change in bias voltage for each PMT with the pulsed light source on. The pulse width and amplitude of the pulse generator was selected to simulate a scintillation photon entering the PMT. The logarithmic-scale graph on the right shows the dark current of the 112 PMTs with the light source off and the bias voltage swept under the same conditions as the graph on the left. 


\subsection{Qualification Methodology}

A method for performing pulse shape discrimination (PSD) in the PRS detectors was developed and optimized, providing a means of differentiating incident neutrons from photons beyond simply using energy-level discrimination to reject photon events. The geometric configuration inside the test box used for PSD development is shown in Figure 12.

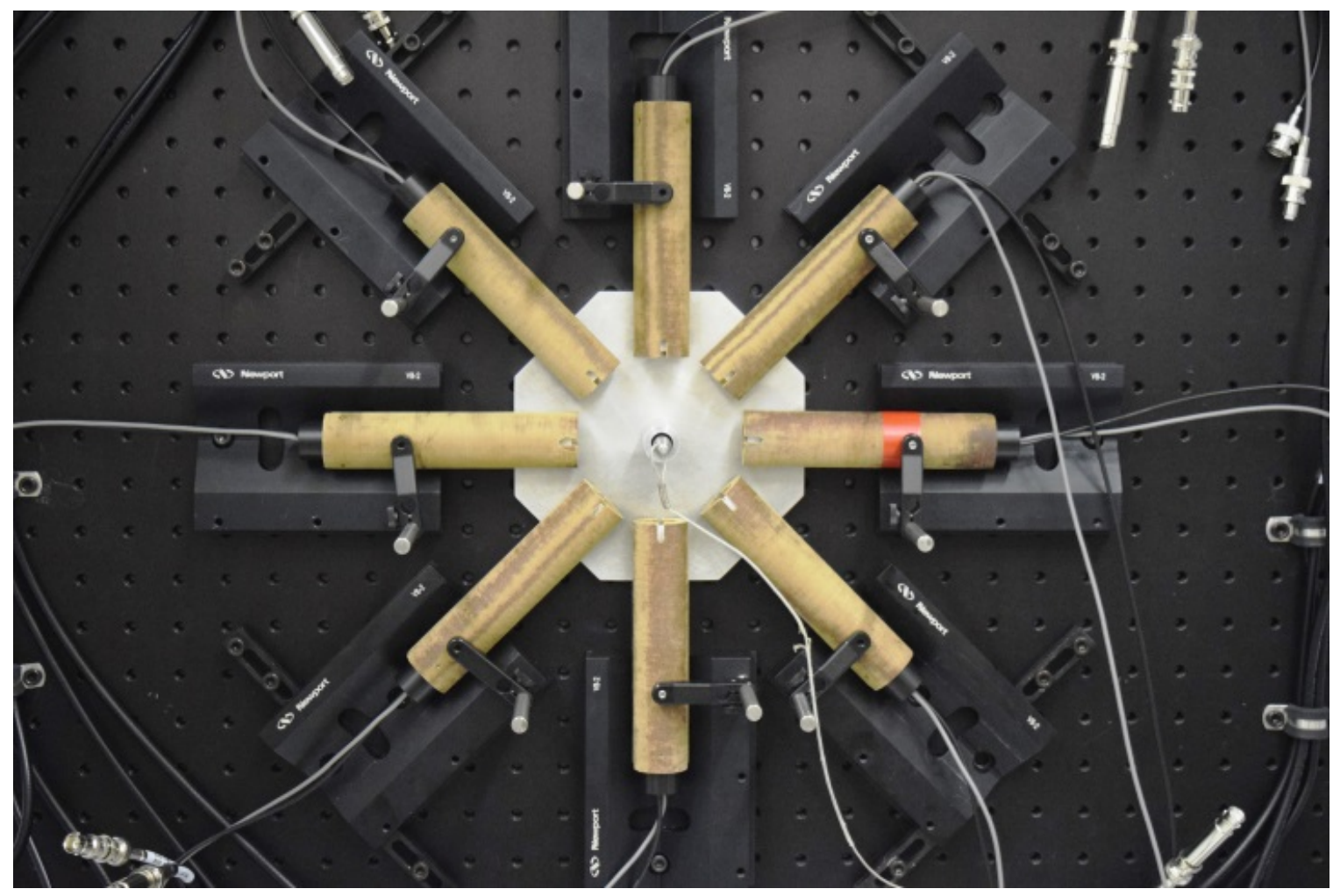

Figure 11 A set of PRS/PMT assemblies configured around a ${ }^{252} \mathrm{Cf}$ source inside Test Station \#2, to offer evenly distributed neutron flux for PSD optimization and detector-to-detector normalization.

Time and amplitude information were also persevered to offer additional PSD algorithm parameters if needed. The algorithm developed for PSD implantation is a charge integration technique using a two-dimensional look up table (LUT). This allows complex neutron and photon regions to be defined in PSD parameter space. The method was implemented in a Struck digitizer (Model SIS3316, 16-channel, 14-bit, $250 \mathrm{MS} / \mathrm{s}$ ) which allows for channel-specific LUT definitions designed for operational conditions where PSD mapping is not identical from detector to detector. The overall methodology used for PSD LUT generation, as well as the data collected per process, is shown in Figure 12. 

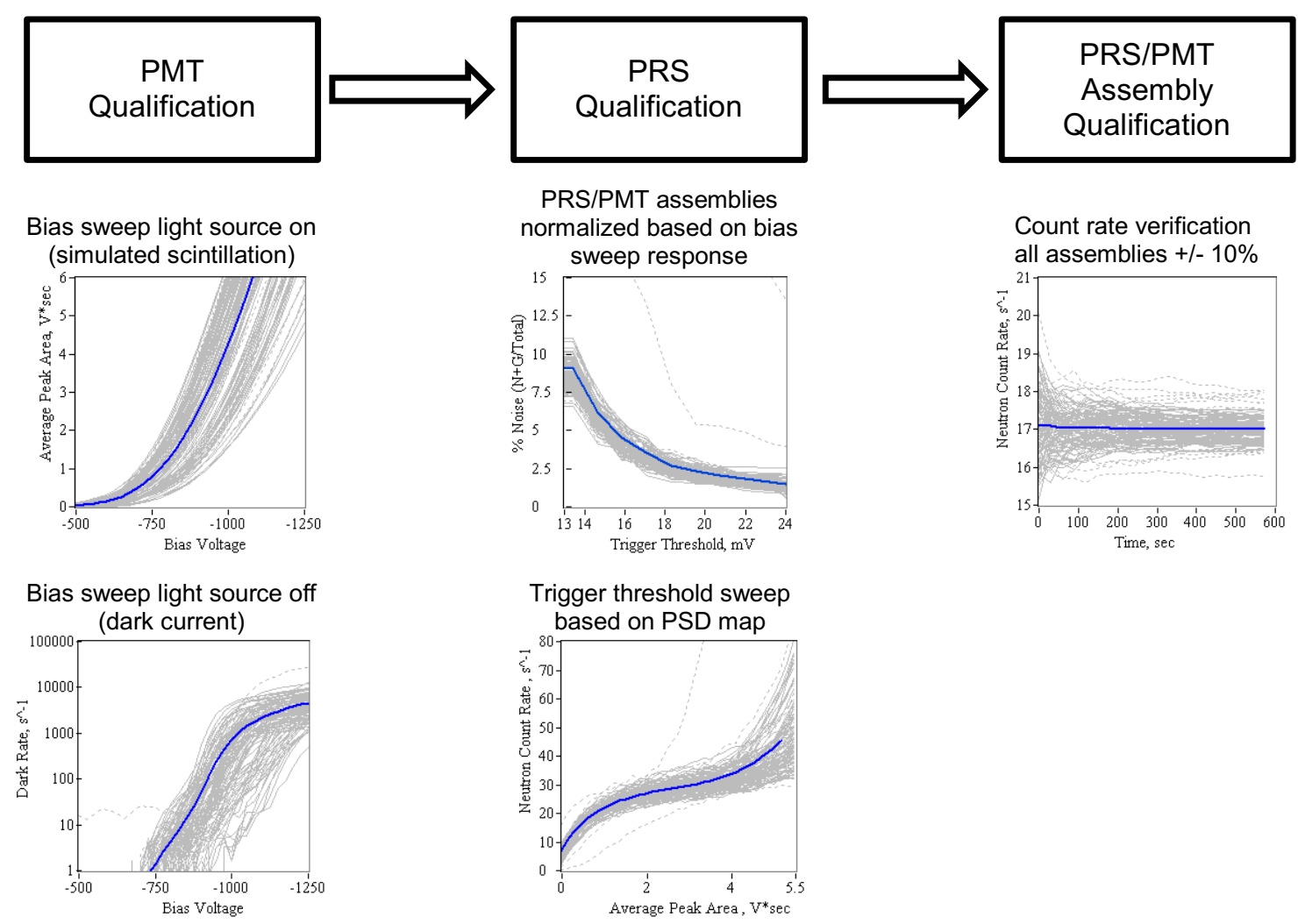

Figure 12 Overall qualification methodology and collected data per process for each PRS, PMT, and PRS/PMT assembly.

Pulses are digitized over a 2-microsecond window. There is a 760-nanosecond-wide region prior to the peak which is used to determine the average baseline voltage. The digitized pulse is divided into two regions for charge integration: an 80-nanosecond region that captures the peak of the pulse followed immediately by a 760-nanosecondwide tail region. The PSD ratio is calculated by taking the baseline subtracted ratio of the peak region to the tail region. A schematic demonstrating the baseline, peak, and tailintegration regions for a digitized pulse is shown in Figure 13. 


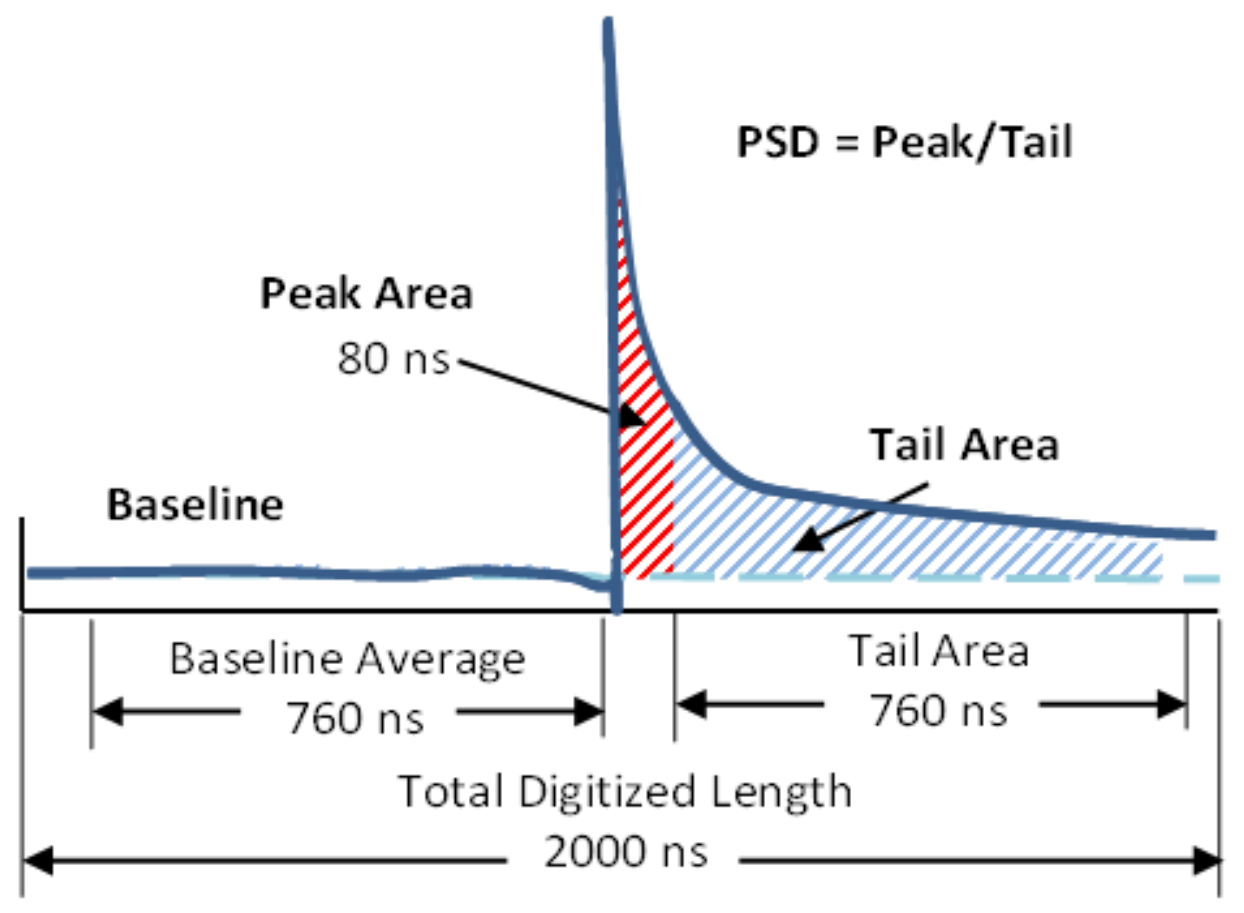

Figure 13 A schematic demonstrating how the PSD value is calculated from a digitized detector pulse (the pulse has been inverted for display).

Neutrons will have a PSD ratio that trends toward 0.5 Volt*seconds, meaning the tail area is about half as large as the peak area. Gammas do not have a tail so the PSD ratio will be centered on zero. Negative PSD ratios are possible if the baseline is perturbed due to noise or a very long lived neutron tail, hence negative ratios will be rejected based on this understanding. Based on using a ${ }^{252} \mathrm{Cf}$ source, each detector was mapped using the PSD method described above and plotted using newly developed acquisition and analysis software. The results of this overall detector PSD mapping technique are shown in Figure 14. 


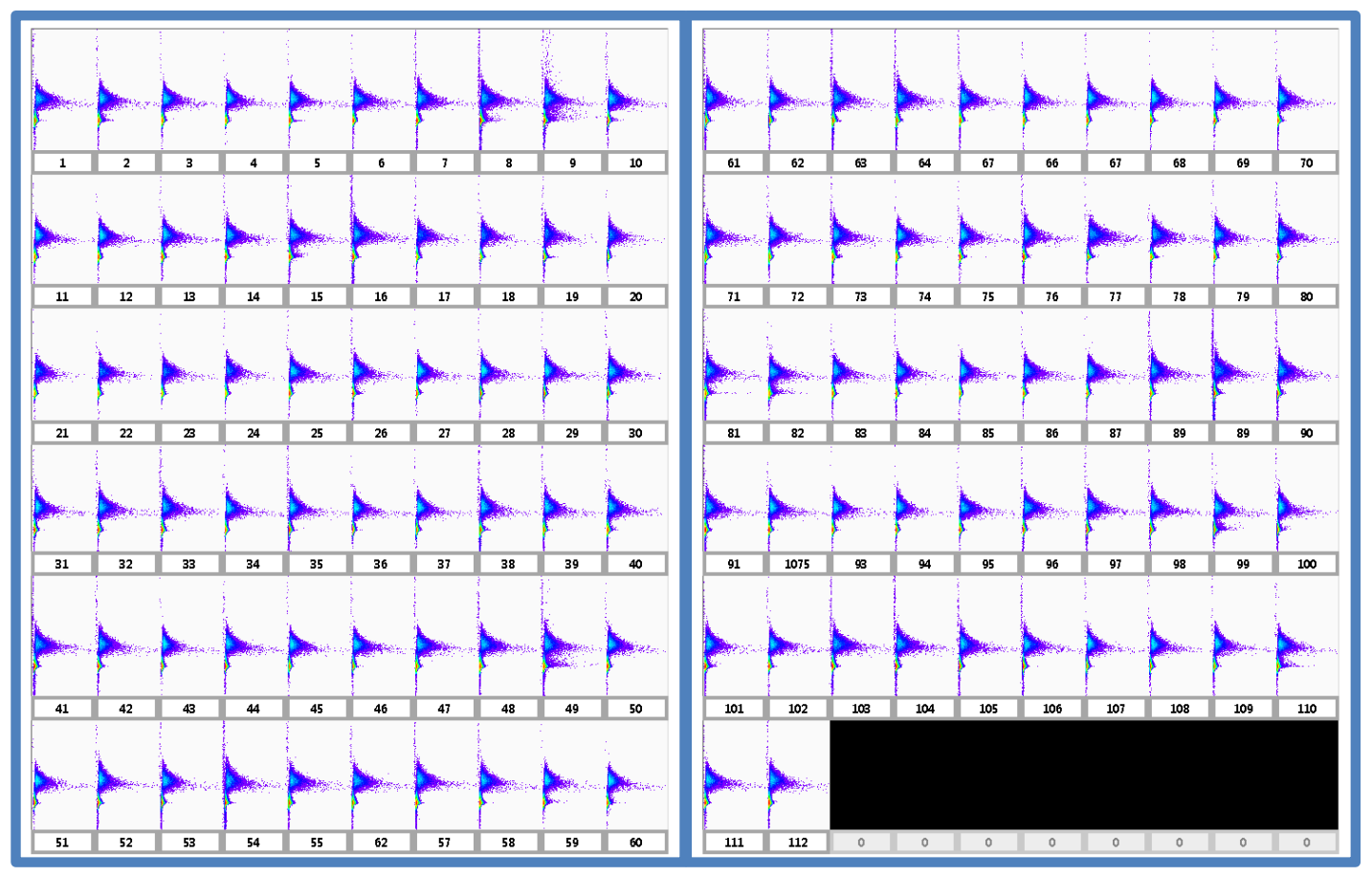

Figure 14 Individual PSD maps assigned per detector assembly based on PMT and PRS response using a ${ }^{252} \mathrm{Cf}$ source.

\section{DATA ANALYSIS / VISUALIZATION SOFTWARE}

A prototype software application was developed for the time-dependent analysis and visualization of data acquired by the FMMS. The application is ready for initial testing of the FMMS. Revision 0.1 of the analysis and visualization software was developed for operation on a Microsoft Windows operating system in C\# and XAML languages. National Instruments analysis libraries were used to insure seamless integration with the FMMS data acquisition software written in the National Instruments LabVIEW development environment. The application was written in C\# and XAML to allow for future revisions to be ported to Apple's macOS, iOS, and Google's Android operating systems. A screen shot of the application is provided in Figure 15. The software package requires both a data set from the FMMS data acquisition system (DAS) and a corresponding time-dependent power profile from the TREAT reactor. The FMMS data files are written in the Technical Data Management Streaming (.TDMS) file format. This is a binary format that allows high speed streaming and searching while providing a very small disk footprint. Revision 0.1 of the application reports neutron signal intensity. Once initial testing is complete the mass coupling factor will be determined experimentally, allowing for future revisions of the software to report values in units of fissioning mass. 


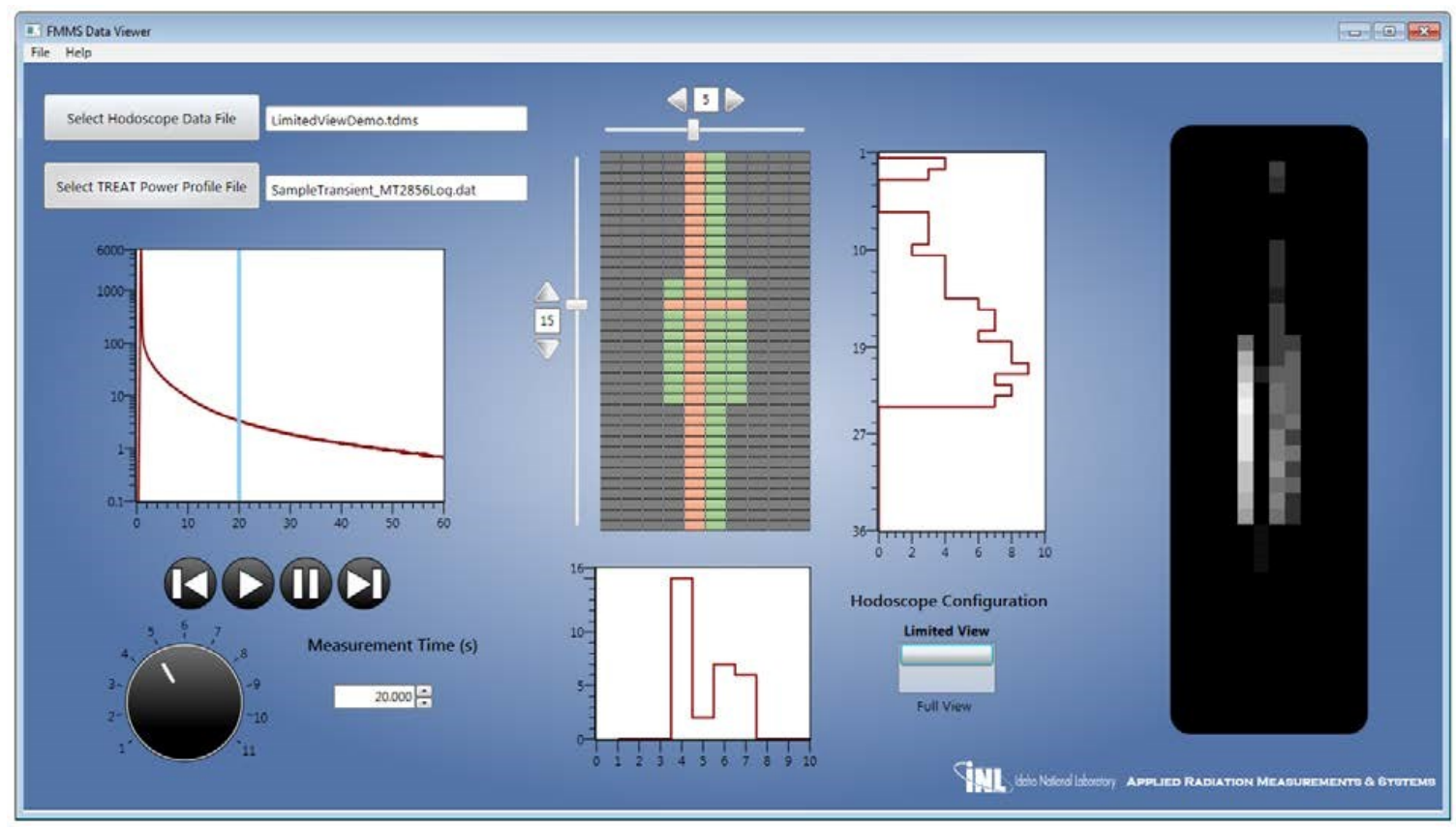

Figure 15 A screenshot of the FMMS Data Viewer application.

Visualization functionality is divided into two subsections of the user interface. The first section, expanded in Figure 16, contains the TREAT reactor power profile plot and playback timing controls. The red line in the plot indicates the reactor power as a function of time during the transient measurement period. The vertical blue line in this plot indicates the current measurement time for which data is visualized. The current time is also displayed in the numerical text box which also allows the user to advance directly to a specific measurement time. Visualization can progress automatically or be manually step-advanced in time using the four playback control buttons. The playback speed and step size is controlled with the knob selector. 


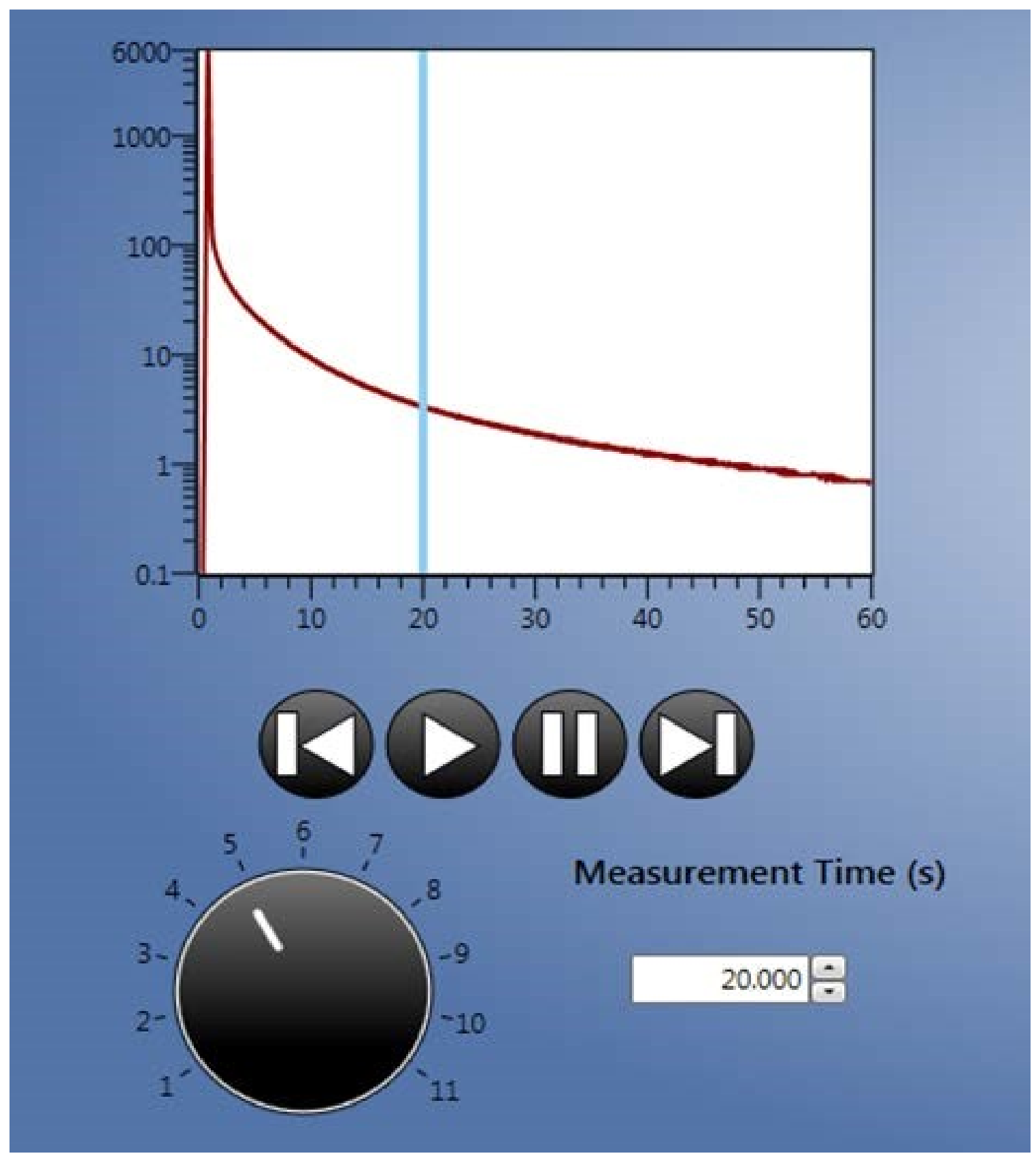

Figure 16 An expanded view of the TREAT reactor power profile plot and data playback controls section of the FMMS Data Viewer user interface.

The second major section of the user interface contains the 1- and 2-dimensional visualization components of the application. An expanded view of this section is provided in Figure 17. A column and row selector grid allows the user to view individual column data (plotted to the right of the grid) or row data (plotted below the grid). The user can use the vertical and horizontal sliders and arrow buttons change the selection or enter values directly into the corresponding numerical text boxes. A switch is provided to shift between limited and full view hodoscope configurations to limit the selector grid to 
the hodoscope channels available in the data set. To the right of the 1-D visualization components is a 2-D visualization of the entire FMMS data set that uses a grey or color scale to indicate signal intensity. All visualization components are updated using the measurement time and playback controls of the previous section.

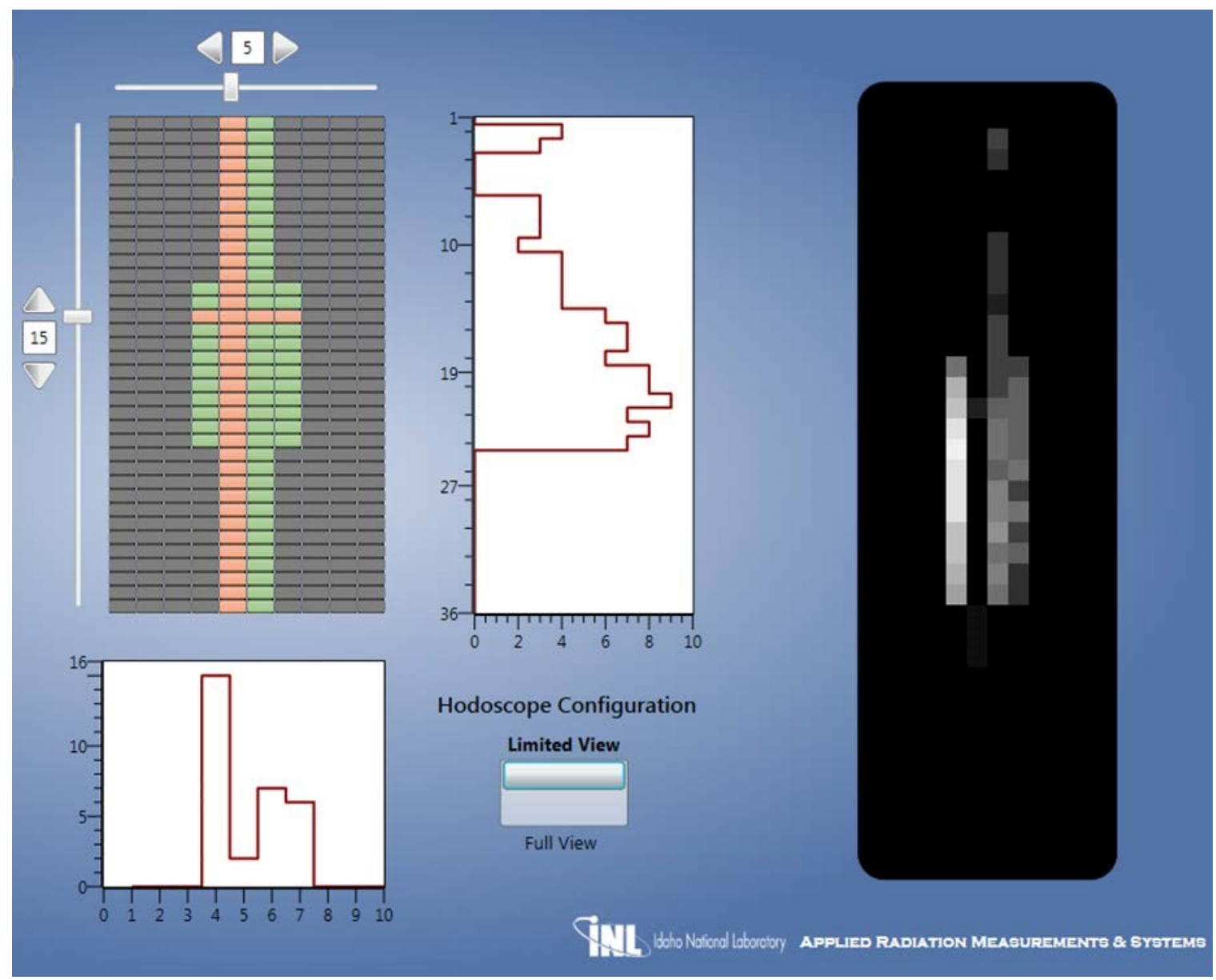

Figure 17 An expanded view of the 1-D and 2-D visualization sections of the FMMS Data Viewer user interface.

\section{FMMS INSTALLATION AT TREAT}

Fuel motion monitoring system engineers, working alongside TREAT Nuclear Operations technicians, installed the limited-view (LV) 96-channel hodoscope during the week of July 14, 2017. The system was designed and assembled at the RSDL laboratory over the course of FY2016/17. The system was then was disassembled, packaged, and transported to TREAT, seen in Figure 18 and Figure 19. The PRS detector assemblies were installed into the light-tight cabinet and the location of each assembly was recorded (row and column location). The detectors were installed into the middle of column four (12), the entire length of columns five and six (36 each), and the middle of column seven (12) for a total of 96 recordable data channels. There were 192 cables that required labeling prior to deployment that were installed and routed through the light-tight cabinet to the adjacent DAS cabinet. Step-by-step installation photos are shown in Figure 18 
through Figure 23. A single CAT-5 communication cable was installed between the TREAT Hodoscope Room and the DAS, to offer temporary local remote operation during the ring-out phase of initial FMMS operations.

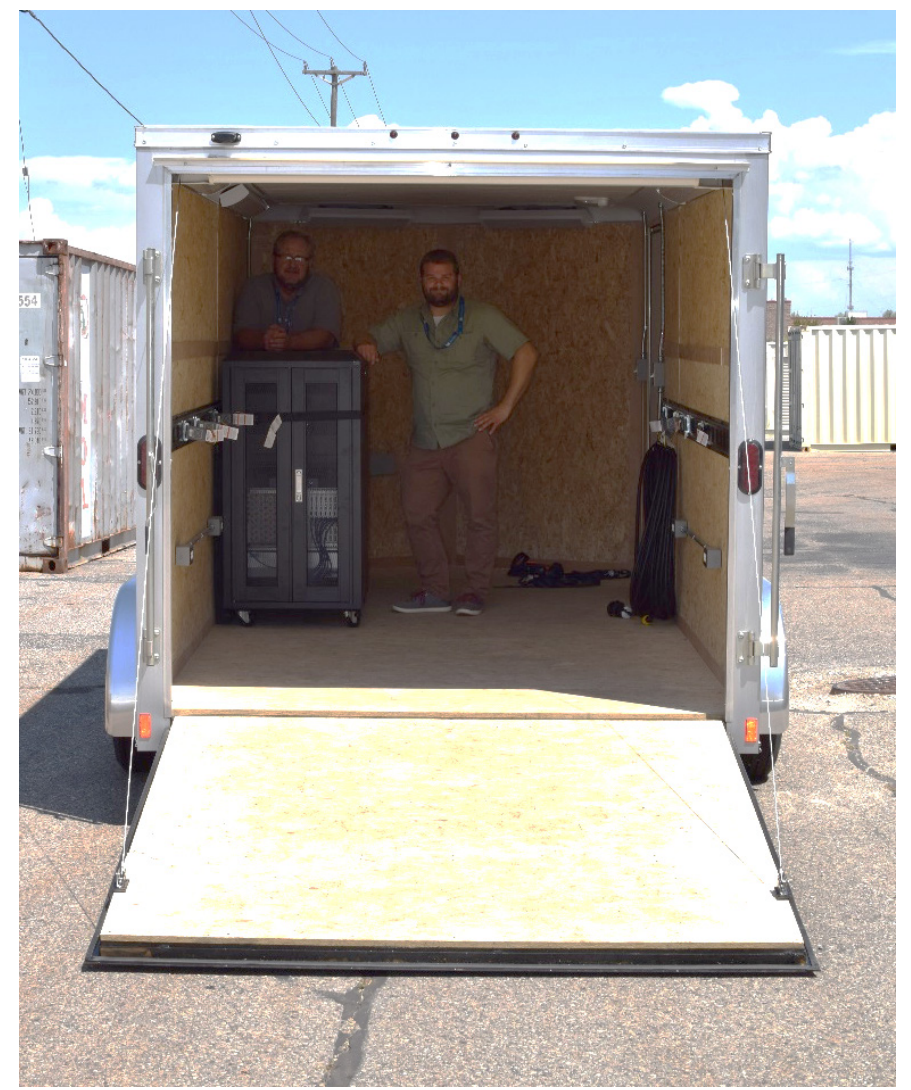

Figure 18 FMMS engineers load and secure the TREAT hodoscope for shipment.

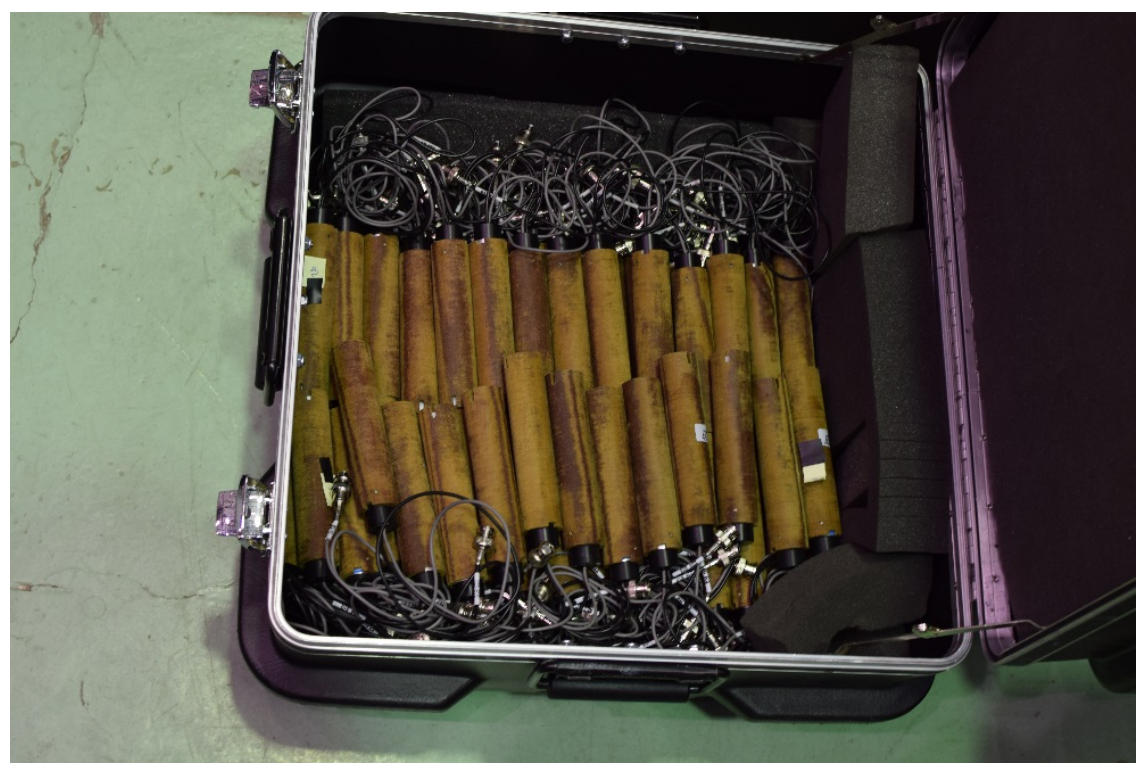

Figure 19 PRS/PMT assemblies ready for installation into the hodoscope at TREAT 


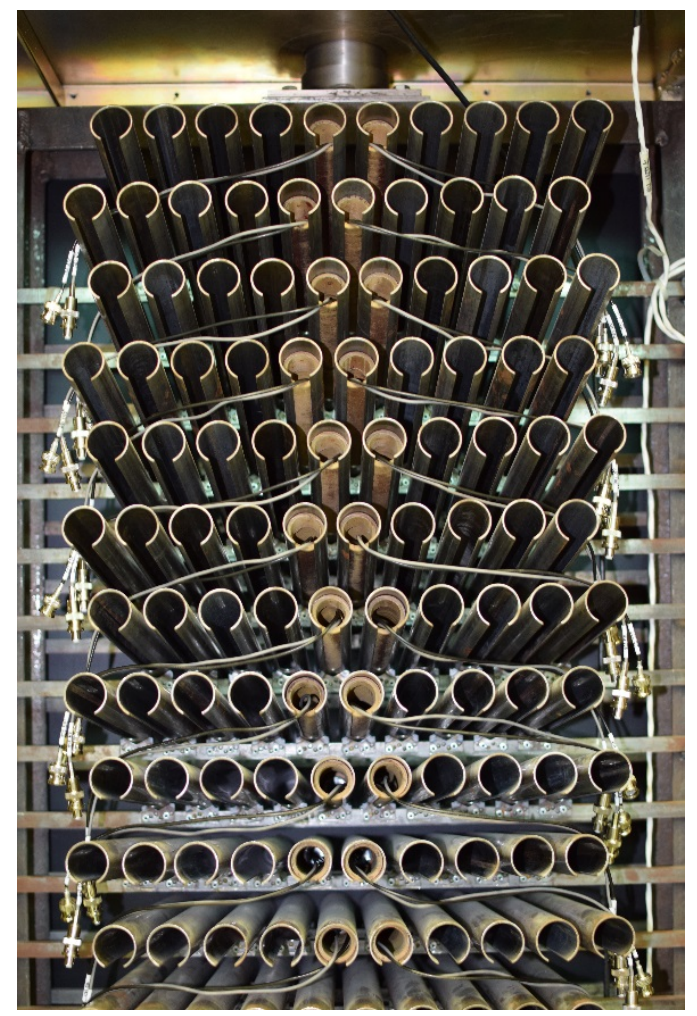

Figure 20 A view inside the light-tight PRS detector cabinet on the hodoscope at the TREAT north port. Detector assemblies are aligned with rear collimator channels.

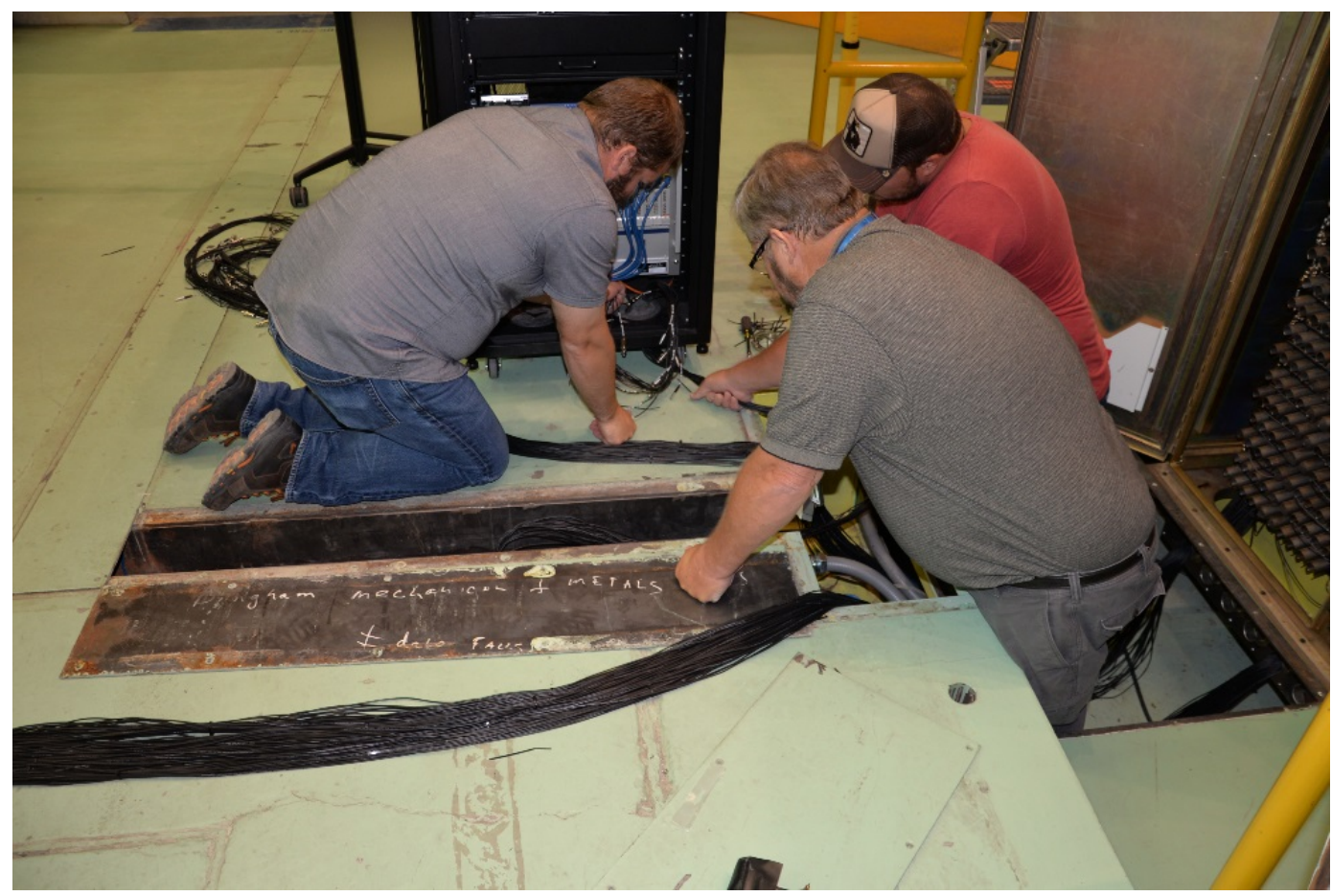

Figure 21 FMMS engineers and a TREAT Instrumentation and Control technician work side-by-side to ensure proper cable routing and dressing during the $L V$ FMMS installation. 


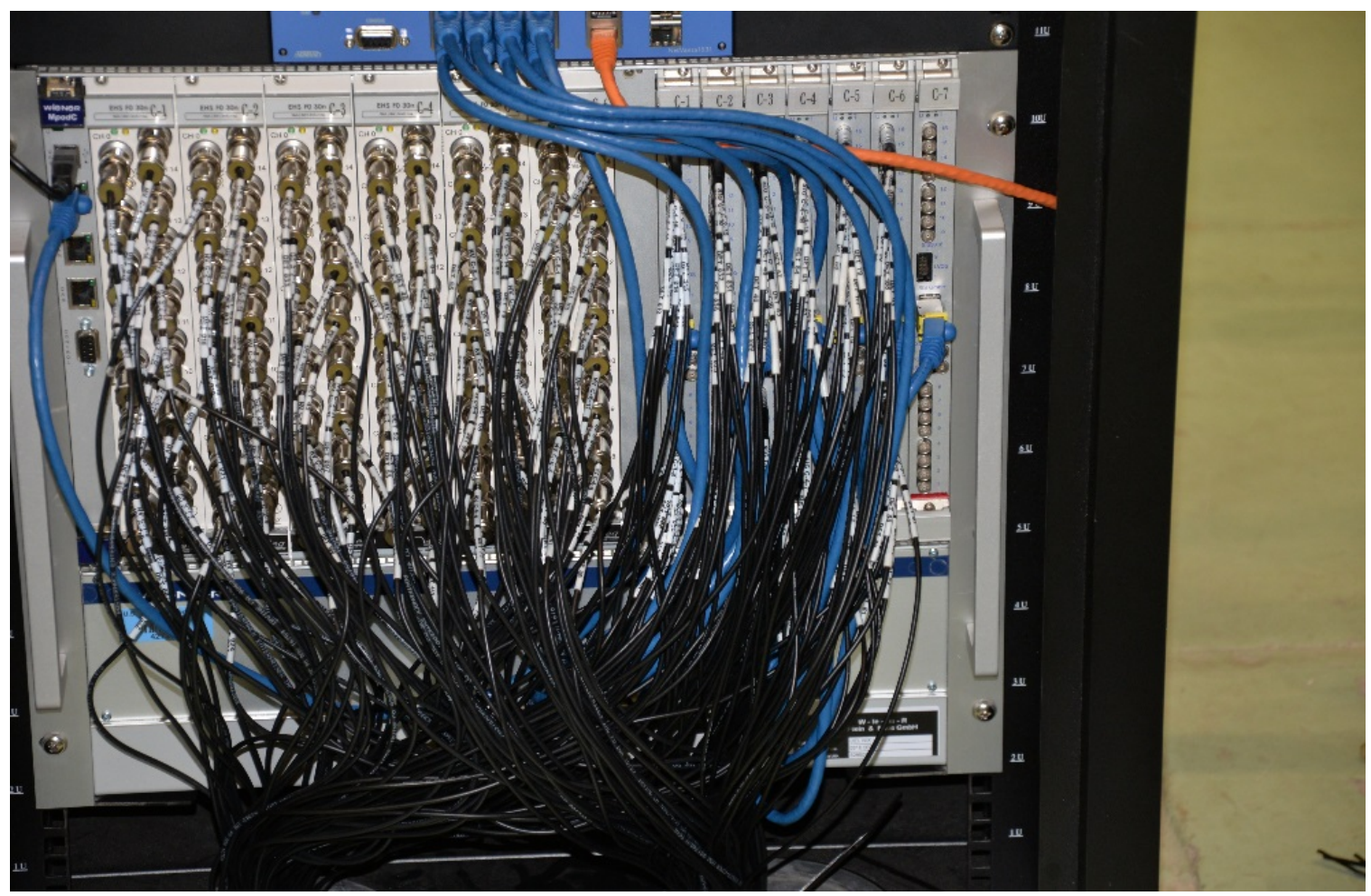

Figure 22 A view of the final connections made to the FMMS DAS at TREAT. 


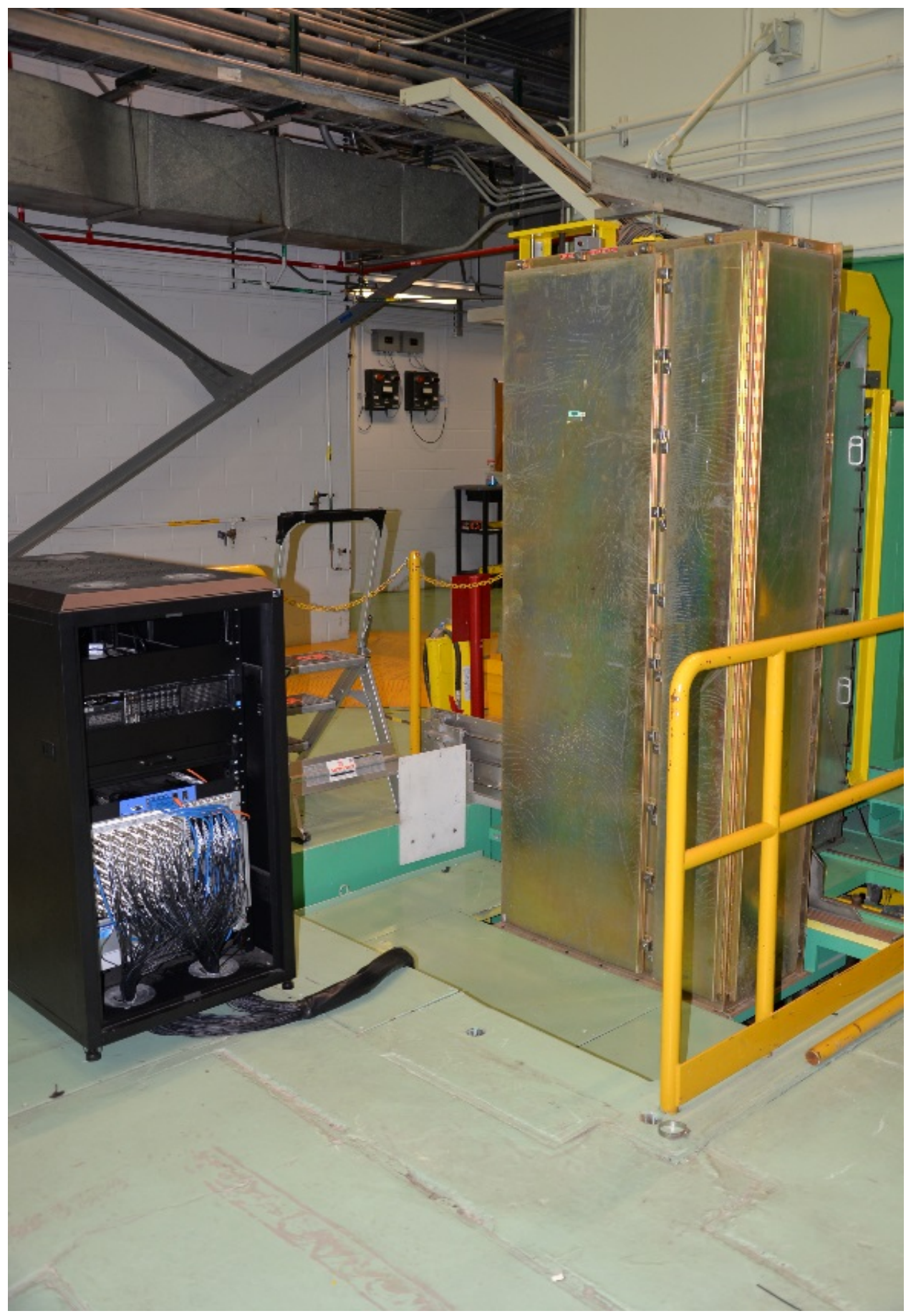

Figure 23 Installation of the DAS, ready for testing. 


\section{ALTERNATIVE DETECTORS}

In FY2017 significant progress was made towards developing a replacement for the original PRS buttons, to support deployment of a full 360 channels of PRS detectors. Work was also performed on initial check out of several spare Reuter-Stokes (model RSP5-0808-204) PRPCs.

\subsection{New PRS Materials}

An effort with made to investigate several fast neutron scintillation materials to possibly replace the original detectors currently being used in the limited-view FMMS. Several detector-material producers, both commercial and research based, were contacted and asked to manufacture materials with the same form factor as the original $\mathrm{ZnS}(\mathrm{Ag})$ wafers. FMMS engineers then took these samples and machined and polished them to achieve the correct final form factor for evaluation. Work was also performed at INL to explore inhouse manufacturing methods for creating $\mathrm{ZnS}(\mathrm{Ag})$ wafers, using casting methods with various liquid epoxies and acrylics that were mixed with a powdered form of $\mathrm{ZnS}(\mathrm{Ag})$ (Eljen Technologies EJ-500). This proved to be the most successful method. Some of the various materials evaluated in this effort are shown in Figure 24.

Using an "ideal" optical output PRS reference button recovered from TREAT, FMMS engineers we able to experiment with various mixtures to obtain a detector that had $75 \%$ of the optical output of the reference button, which put the INL manufactured button in the usable category using the standard down selection method developed earlier in 2016. The light output of the various materials evaluated in this effort are shown in Figure 25. Further work is needed in FY2018 to examine the PSD performance of these materials. 


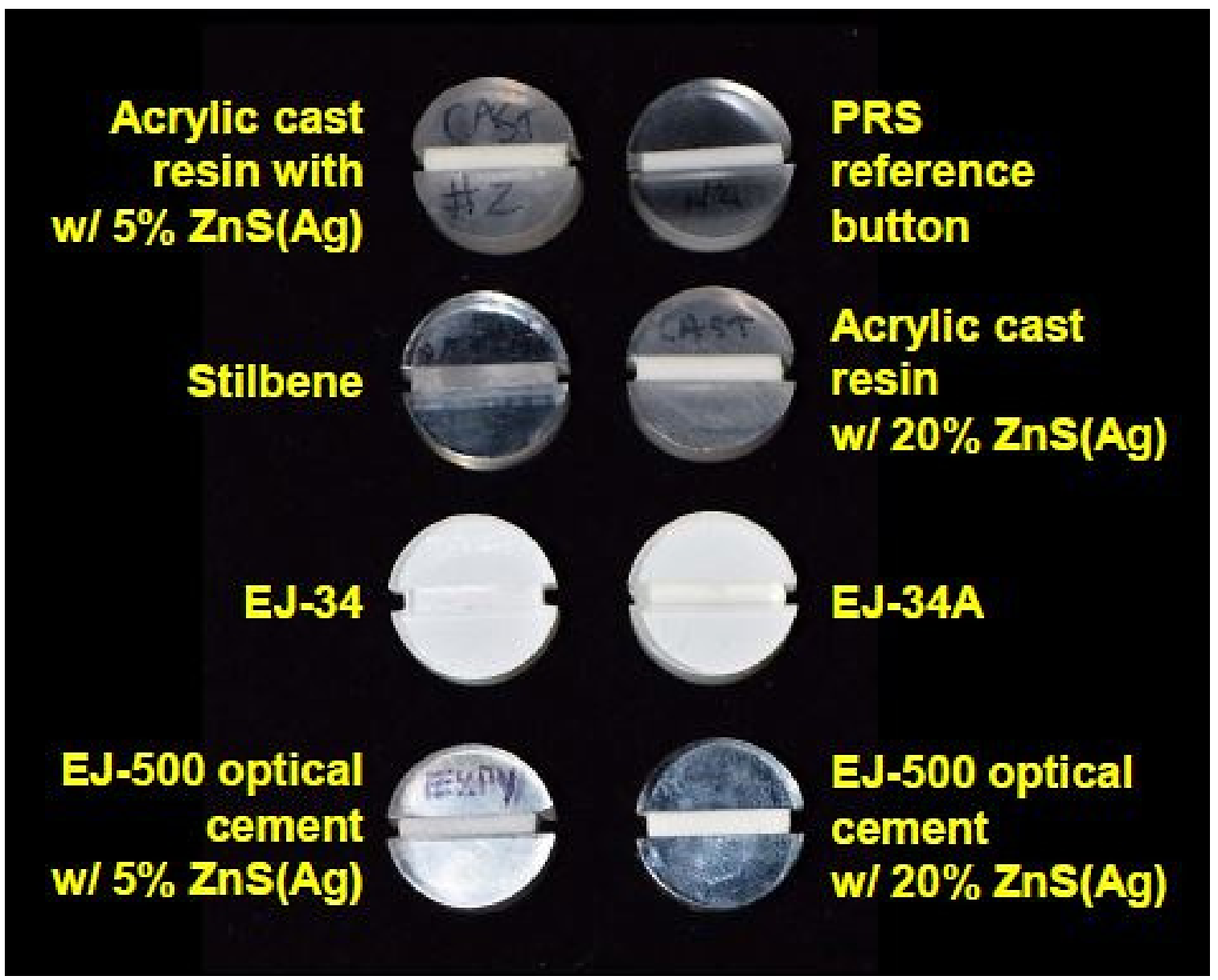

Figure 24 Various scintillation materials used in the search for a replacement for the PRS detectors. 


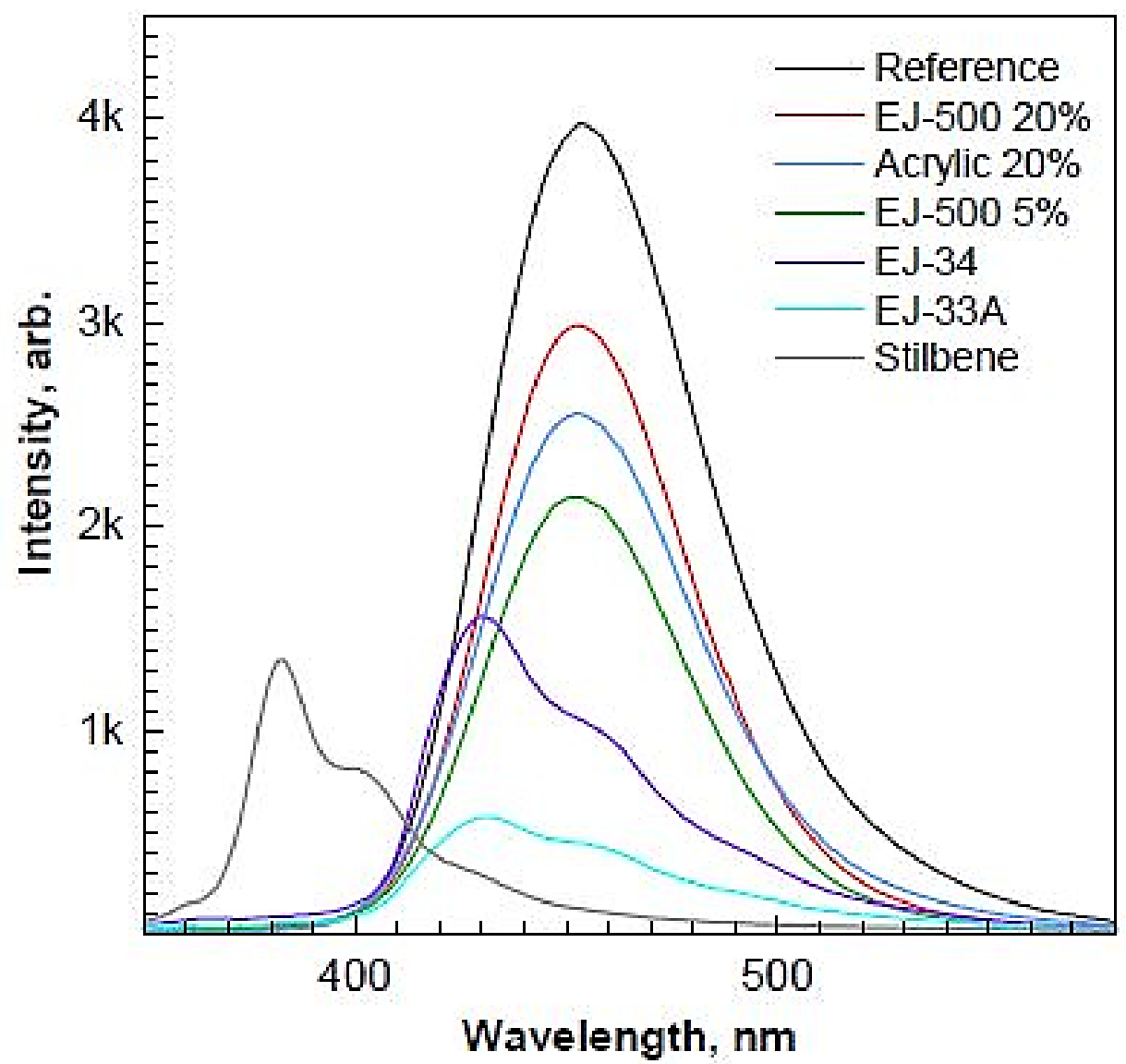

Figure 25 Optical output response of various materials tested, using a 300-nm light source for excitation. Note the $20 \% \mathrm{ZnS}(\mathrm{Ag})$ mixture in optical epoxy demonstrates the best result of the new candidate sensors.

\subsection{Proportional Counters}

X-ray images (Figure 26) were taken to gain a better understanding of the inner workings of individual PRPCs. A data acquisition system was then assembled to perform initial testing on the PRPCs. Care had to be taken while biasing the detectors with high voltage so that arcing did not occur, since the documented turn on bias voltage is listed as $+5,600$ $\mathrm{V}$. The experimental set up included an acrylic shield in which each detector under test was placed, this prevented the possibility of electrical shock and provided a means of electrical isolation from grounding sources that might cause arcing. The original signal conditioning electronics were bypassed and data was acquired using a digital oscilloscope. The output signals from the raw PRPCs matched typical waveforms from this style of fast neutron detector. The experimental set up used to perform the PRPC check out is shown in Figure 27 and Figure 28. Further work will be needed in FY2018 to develop an operation plan for working with these detectors, followed by testing with a neutron source and evaluation of the state of health of the facility infrastructure at TREAT. 


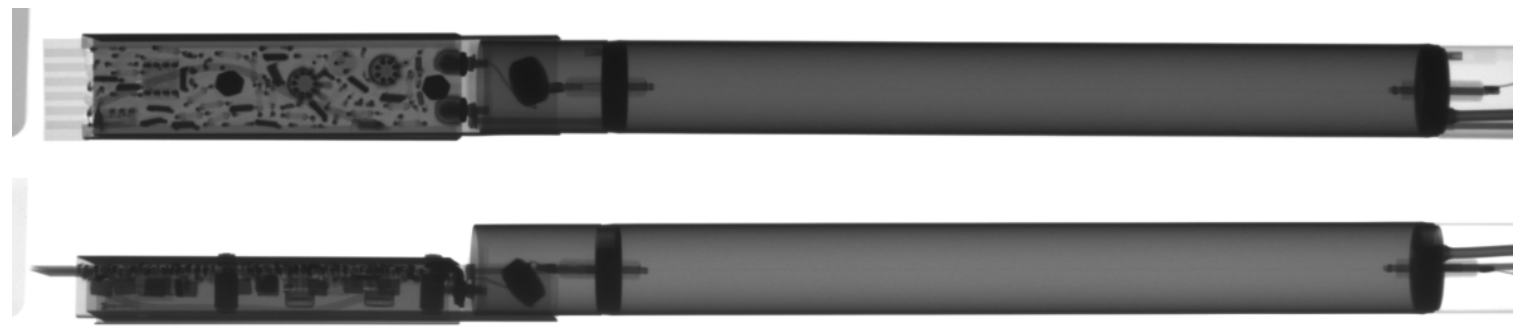

Figure 26 X-ray image of Reuter-Stokes (model RS-P5-0808-204) PRPC.

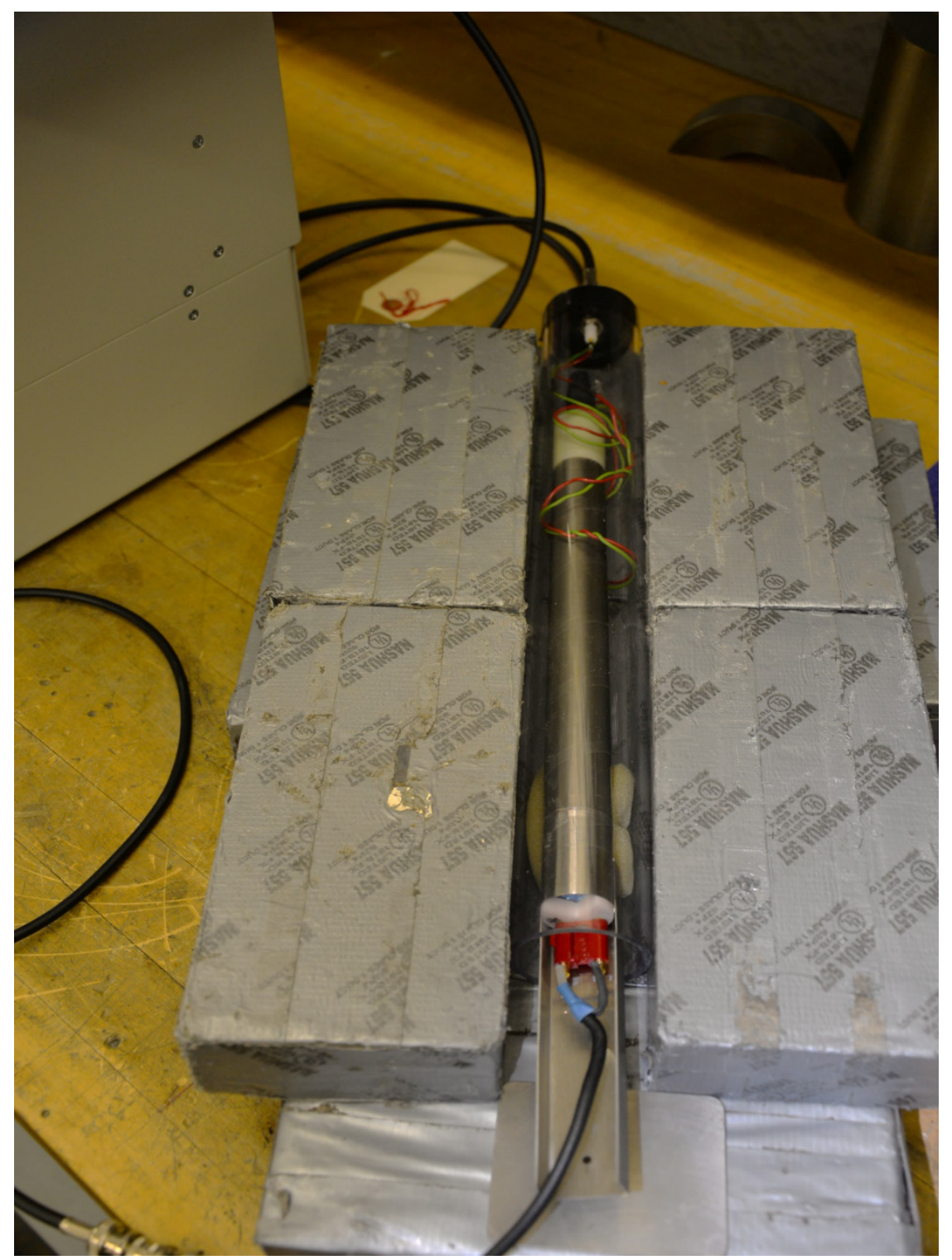

Figure 27 Reuter-Stokes (model RS-P5-0808-204) under test inside acrylic HV shield. 


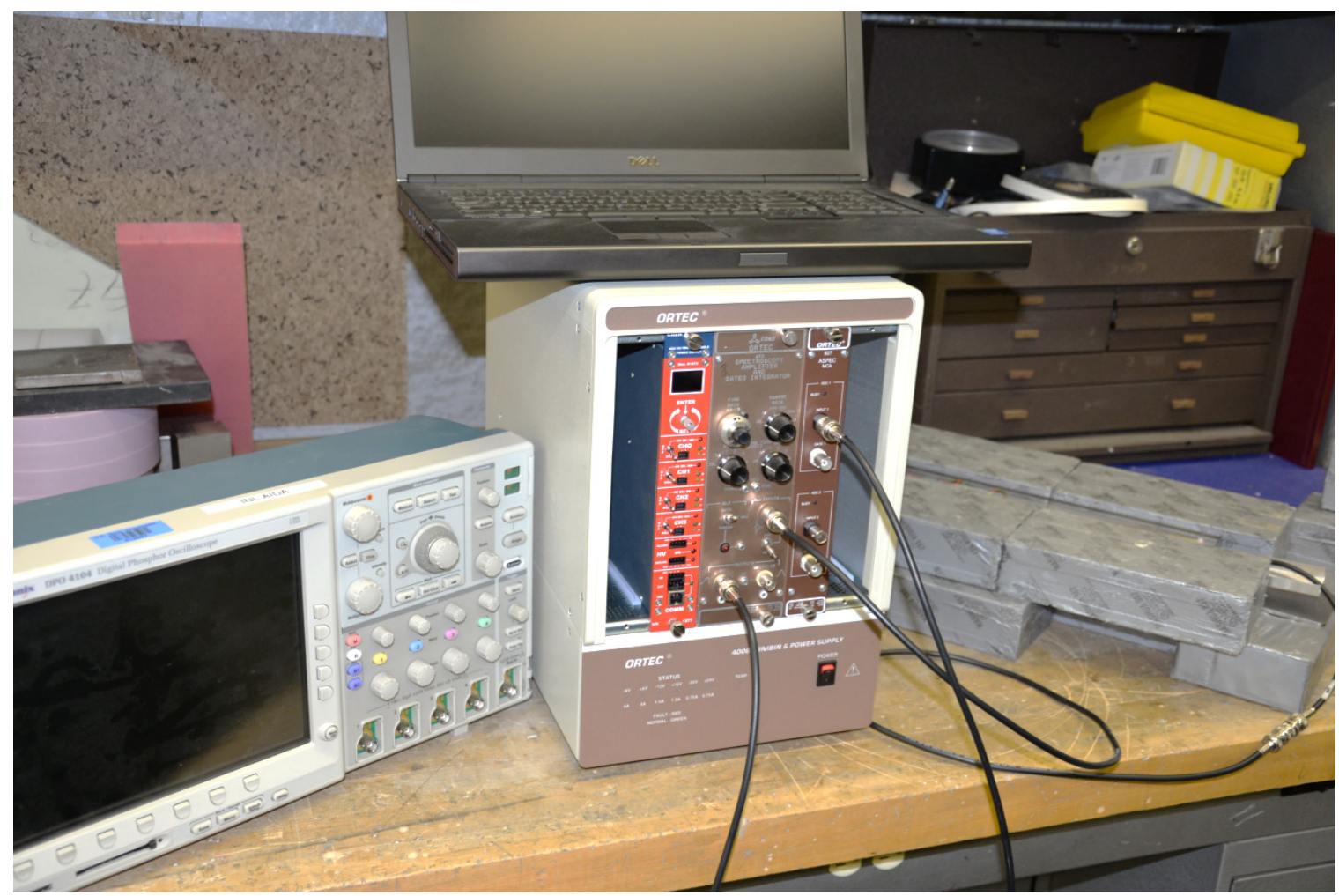

Figure 28 Initial experimental set up for PRPC testing. Note the use of the $+8 \mathrm{kV}$, four-channel high voltage supply since the bias turn on voltage was stated as $+\mathbf{5 , 6 0 0}$ V. 


\section{REFERENCES}

Watson, S. M., et al., "A Multi-Purpose Test Station to Characterize Fast Neutron Detectors for the TREAT Fuel Motion Monitoring System," Conf. Proc. IEEE Nucl. Sci. Symp., Atlanta, Ga., Oct. 21-28 (2017) Submitted.

Hix, J. D., et al., "Evaluation, Refurbishment, and Characterization of Fast Neutron Scintillators for the TREAT Fuel Motion Monitoring System," Conf. Proc. IEEE Nucl. Sci. Symp., Atlanta, Ga., Oct. 21-28 (2017) Submitted.

Johnson, J. T., et al., "Optimization of a Fast Neutron Scintillator for Real-Time Pulse Shape Discrimination in the Transient Reactor Test Facility (TREAT) Hodoscope," Conf. Proc. IEEE Nucl. Sci. Symp., Strasbourg, France, Oct. 29 Nov. 5 (2016) In Press.

Thompson, S. J., Townsend, C. H., and Chichester, D. L., "Preliminary Modeling to Support the TREAT Hodoscope Systems for Fuel Motion Monitoring," Conf. Proc. PHYSOR 2016, Sun Valley, Idaho, May 1 - 5 (2016), 426-433.

Chichester, D. L., et al., "The TREAT Fast-Neutron Hodoscope and Plans for Restoring it to Operation," Trans. Amer. Nucl. Soc. 113 (2015) 377-380. 